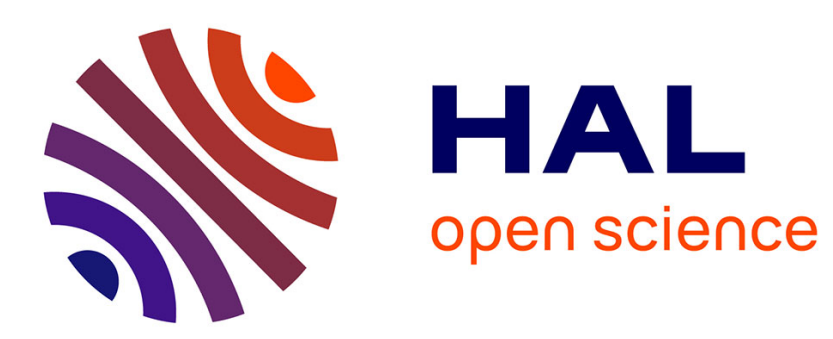

\title{
Reduced dynamics for delayed systems with harmonic or stochastic forcing
}

Jérémie Lefebvre, Axel Hutt, Viktor Leblanc, André Longtin

\section{To cite this version:}

Jérémie Lefebvre, Axel Hutt, Viktor Leblanc, André Longtin. Reduced dynamics for delayed systems with harmonic or stochastic forcing. Chaos: An Interdisciplinary Journal of Nonlinear Science, 2012, 22 (4), pp.043121. 10.1063/1.4760250 . hal-00764986

\section{HAL Id: hal-00764986 https://hal.inria.fr/hal-00764986}

Submitted on 17 Jul 2013

HAL is a multi-disciplinary open access archive for the deposit and dissemination of scientific research documents, whether they are published or not. The documents may come from teaching and research institutions in France or abroad, or from public or private research centers.
L'archive ouverte pluridisciplinaire HAL, est destinée au dépôt et à la diffusion de documents scientifiques de niveau recherche, publiés ou non, émanant des établissements d'enseignement et de recherche français ou étrangers, des laboratoires publics ou privés. 


\title{
Reduced dynamics for delayed systems with harmonic or stochastic forcing.
}

\author{
Jérémie Lefebvre, Axel Hutt, Victor G. LeBlanc and André Longtin
}

(Dated: July 17, 2013)

\begin{abstract}
The analysis of nonlinear delay-differential systems (DDE's) subjected to external forcing is difficult due to the infinite dimensionality of the space in which they evolve. To simplify the analysis of such systems, the present work develops a non-homogeneous center manifold (CM) reduction scheme, which allows the derivation of a time-dependent order parameter equation in finite dimensions. This differential equation captures the major dynamical features of the delayed system. The forcing is assumed to be small compared to the amplitude of the autonomous system, in order to cause only small variations of the fixed points and of the autonomous CM. The time-dependent CM is shown to satisfy a non-homogeneous partial differential equation. We first briefly review CM theory for DDE's. Then we show, for the general scalar case, how an ansatz that separates the CM into one for the autonomous problem plus an additional time-dependent order-two correction leads to satisfying results. The paper then details the application to a transcritical bifurcation subjected to single or multiple periodic forcings. The validity limits of the reduction scheme are also highlighted. Finally, we characterize the specific case of additive stochastic driving of the transcritical bifurcation, where additive white noise shifts the mode of the probability density function of the state variable to larger amplitudes.
\end{abstract}

PACS numbers:

Delayed feedback systems have a widespread use accross the engineering, physical and biological sciences. In their applications, these are often subjected to temporally varying forcing to implement the active role played by external influences on the dynamics. However, due to the complexity of infinite dimensional systems, the way additive temporal fluctuations interact with retarded dynamics is far from understood. In particular, a statement on the exact role played by non-homogeneous components on the stability of solutions in delayed feedback models is still missing. Here, the authors propose a novel approach to handle that problem using center manifold theory formulated for delay equations, where an explicit time-dependence is taken into account. Their method makes possible to capture the effect of forcing on the stability of an delayed feedback system in the vicinity of a bifurcation, and serves as new strategy to highlight novel non-linear phenomena.

\section{INTRODUCTION}

Delayed feedback plays an important role in numerous areas such as laser dynamics [1, 2], chemical reactions [3], mechanical systems (see [4] for a review) and general feedback control in simple and complex systems [5-7]. It also plays a major role in shaping the behavior of biological systems $[4,8,9]$. It is particularly important in neuroscience where feedback loops are a ubiquitous component of neural circuitry. Delayed feedback underlies a wide range of phenomena, from simple reflexes [10] to posture control (see $[11,12]$ for examples with, respectively, one and two delays), neural network dynamics [13], brain waves in health [14, 15] and disease [16] states, and more recently anesthesia [17]. Delays also constitute one of the main mechanisms underlying network synchronization and play a critical role in the stability of activity patterns in spatially extended systems [18-20].

The question of how spatio-temporal forcing interacts with retarded dynamics is an unexplored yet important frontier, presumably because of the mathematical complexity of dynamical analyses of even autonomous retarded dynamics. For example, the exact role of delays in the integration of temporal signals in sensory systems is beginning to receive attention (see e.g. [21]), but much remains to be understood due to the lack of tools that dynamical systems theory provides for the study of the stability of non-homogeneous and/or non-autonomous retarded problems. It is unfortunately still unclear whether to apply e.g. the center manifold theorem, and if so how, let alone compute center manifolds in specific cases for non-autonomous delayed systems. And should one simply append the forcing onto the reduced dynamics of the autonomous system? Advances on this problem would thus greatly enhance the scope of theoretical analyses of delayed systems.

Our paper addresses this lack by developing center manifold reductions in the presence of external forcing. Indeed, the center manifold theorem has proven to be a powerful tool for classifying the behavior of all autonomous 
dynamical systems including retarded ones [22, 23]. It is thus deemed a promising candidate as a tool for nonautonomous retarded system. However, the question whether the center manifold theorem may be extended to non-autonomous delayed problems is currently left unaddressed. There are indications that center manifolds do exist in infinite dimensional non-autonomous dynamical systems [24]. Furthermore, stochastic center manifold theory has been established in non-delayed noisy systems [25-27], and a similar approach has been used in the numerical analysis of non-linear ODEs subject of time-dependent forcing, providing accurate results [28]. These studies provide fruitful avenues to pursue, including the one followed below. To the best of our knowledge, our study is the first to treat analytically the dynamics of delayed system controlled by external driving. Notably, such control is widely applied in various neuroscience fields, such as in neuropsychological experiments in form of flicker experiments [63] or as Deep Brain Stimulation in Parkinson Disease [62, 64], to name a few. The present work fills this gap and illustrates the practical mathematical application by a rather simple but revealing non-linear example.

In particular, our recent work [29] showed how a driven by Gaussian white noise could be analyzed by making an ansatz of separability for the CM into an autonomous part and a time-dependent correction. That work then derived an order parameter equation involving white and colored noises, and a Fokker-Planck analysis with time-scale separation was used to approximate the probability density of the state variable. This revealed interesting shifts of the mode of the density caused by the interaction of additive noise and the delay. The question remains open whether the ansatz is a fruitful approach to the deterministic forcing problem, and whether trajectories are well represented by the resulting reduced dynamics for both the deterministic and stochastic forcing cases. The method is thus put on a firmer footing below and explored in detail for the deterministic case.

We first proceed by a detailed outline the procedure of center manifold reduction in delayed systems, summarizing the detailed discussions of [30-34]; this includes the suspension that allows the unfolding of the bifurcation as the critical parameter changes. The goal of this detailed review is two-fold: 1. provide a goal-oriented and pragmatic description of delayed center manifolds calculations that we haven't found elsewhere, and 2. expose the key steps subject to specific asumptions while adapting the theory to the non-autonomous problem. Based on this, we present a novel theoretical tool to study delayed dynamical systems subject to external forcing, and illustrate its use by studying a simple example. We propose a solution to the presence of non-autonomous contributions in the form of an additive temporal correction to the center manifold computed in the autonomous case. To illustrate the idea, we compute the center manifold explicitly for a driven delayed model with a co-dimension one transcritical bifurcation, which has a quadratic non-linearity. Numerical simulations are then used to show that our approach accurately describes the pathwise dynamics in these systems near these bifurcation points. This is done for different orders of approximation, and for a range of forcing frequencies and amplitudes. The work also addresses the case of multiple driving frequencies, as well as Gaussian white noise. The paper ends with a discussion of the method, its successes and limitations, and gives an outlook onto future work.

\section{CENTER MANIFOLD REDUCTION}

In this Section we briefly review the standard methodology to analyze delay differential equations (DDEs), and the procedure of autonomous center manifold reduction. These subsections provide the basis for our proposed non-autononous center manifold reduction technique, presented in last subsection.

\section{A. Analysis of autonomous DDEs}

Consider the general autonomous scalar delay differential equation,

$$
\begin{array}{rlrl}
\dot{x}(t) & =f(x(t), x(t-\tau), \varepsilon)=L(x(t), x(t-\tau), \varepsilon)+F(x(t), x(t-\tau), \varepsilon) \\
\frac{d \varepsilon}{d t} & = & 0 .
\end{array}
$$

where we operated a suspension by introducing a parameter $\varepsilon$, defined by the distance from the bifurcation. The operator $L$ is a linear function with $L(0,0,0)=0$, and $F$ is a non-linear and sufficiently smooth function, satisfying $F(0,0,0)=D F(0,0,0)=0$, where it is assumed that the Jacobian is calculated with respect to all state variables i.e. 
$u$ and $\varepsilon$. Both linear and non-linear parts of this system may contain delayed components. In this section, we will introduce the notation and define the material required to perform the rather standard center manifold reduction of delayed functional differential equations. Reviews and in depth discussions of this method can be found in [30, 31, 34, 35].

In order to consider solutions $x(t)$ of $(2)$ for $t \geq 0$, one needs a complete description of the initial value problem, corresponding to the retarded dynamics on the interval $[-\tau, 0]$. It is therefore imperative to consider Eq. $(2)$ in an appropriate phase space. To take into account the continuous dependence of the flow $x(t)$ on the retarded dynamics, it is convenient to introduce the parameter $\theta$ with $-\tau \leq \theta \leq 0$ and a new variable $z_{t}(\theta) \in \mathbb{R}^{2}$, so that $z_{t}(\theta) \equiv(x(t+\theta), \varepsilon)^{T}$. Based on this definition, the appropriate phase space can be shown to be the Banach space of continuous maps $\mathcal{C} \equiv C([-\tau, 0], \mathbb{R} \times \mathbb{R})[31,34]$. Then, the linear operator $L\left[z_{t}\right]$ may be written as [30]

$$
L\left[z_{t}\right]=\int_{-\tau}^{0} d \eta[\theta] z_{t}(\theta)
$$

with $d \eta[\theta]=\omega(\theta) d \theta$ and the density function $\omega(\theta)$; this representation follows from Riesz's Theorem. For the scalar DDE and a single parameter, $\omega(\theta)$ is a $2 \times 2$-matrix. The initial system in Eq. (2) may thus be written in $\mathcal{C}$ as a infinite-dimensional ODE,

$$
\frac{d}{d t} z_{t}(\theta)=\mathcal{A}\left(z_{t}(\theta)\right)+X_{o} F\left(z_{t}(\theta)\right)
$$

where the infinitesimal generator $\mathcal{A}$ is defined by $[31,34]$

$$
\mathcal{A}\left(z_{t}(\theta)\right) \equiv \frac{\partial z_{t}(\theta)}{\partial \theta}+X_{o}\left(L\left[z_{t}\right]-\left.\frac{\partial z_{t}(\theta)}{\partial \theta}\right|_{\theta=0}\right) .
$$

The connection function $X_{o}(\theta)$ allows the simultaneous treatment of the cases $\tau \leq \theta<0$ and $\theta=0$. It is defined ad hoc as $X_{o}(\theta)=0, \forall-\tau \leq \theta<0$ and $X_{o}(\theta)=\mathbb{I}_{2}$ for $\theta=0$, where $\mathbb{I}_{2}$ is the $2 \times 2$ identity matrix. Hence, the dynamics of $(x(t+\theta), \varepsilon)^{T}=\bar{z}_{t}(\theta)$ is governed by the infinite-dimensional ODE of Eq. (3) appropriately defined in $\mathcal{C}$ and parameterized by $\theta$.

\section{B. Spectral analysis and phase space decomposition}

To reduce the system on the center manifold, we need to investigate the spectrum $\sigma(\mathcal{A})=\{\lambda \in \mathbb{C} \mid \Delta(\lambda)=0\}$ of the linear operator $\mathcal{A}$ in Eq.(4). Let us define the linearly independent set $\Phi(\theta)=\left[\phi_{1}(\theta), \phi_{2}(\theta), \ldots\right]$ of eigenvectors associated with the Lyapunov exponents, or eigenvalues $\lambda_{i} \in \sigma(\mathcal{A})$. Under some technical assumptions (see section 7.8 of [30]), $\Phi(\theta)$ constitutes a basis for the phase space $\mathcal{C}$. Let us also define the adjoint basis $\Psi(s)=\left[\psi_{1}(s), \psi_{2}(s), \ldots\right]$, where the $\psi_{i}(s)$ are eigenvectors of the adjoint linear problem. The bases $\Phi(\theta)$ and $\Psi(s)$ are such that, by definition,

$$
(\Psi, \Phi)(\theta)=\mathbb{I}
$$

where we have introduced the bilinear form

$$
(a(\theta), b(\theta)) \equiv a(0) b(0)-\int_{-\tau}^{0} \int_{0}^{\theta} a(\xi-\theta) b(\xi) d \xi[d \eta(\theta)] .
$$

of two arbitrary complex functions $a, b$.

The decomposition of the spectrum is fundamental for determining the stable and unstable manifolds in the dynamics of Eq. (3) and thus characterizing its instabilities. In the vicinity of an instability, we might assume, without loss of generality, that a finite number of Lyapunov exponents cross the imaginary axis while changing a 
control parameter, while all other exponents remain confined to the left-hand plane i.e. the spectrum exhibits a finite set of critical eigenvalues with $\lambda=0$ leading to $\operatorname{dim}(U)<\infty$. These bifurcating, or unstable exponents near the transition point introduce a very slow time scale, while the stable components relax much faster to their steady state. As a consequence, after a sufficiently long time, the dynamics of the system is essentially determined by the slow unstable modes $[23,30]$.

If one considers the spectral subset $\sigma_{\mathcal{U}}(\mathcal{A}) \equiv\{\lambda \in \mathbb{C} \mid \operatorname{Re}(\lambda)=0\}$, phase space may be decomposed as $\mathcal{C}=\mathcal{U}+\mathcal{S}$, where $\mathcal{S}=\overline{\mathcal{U}}$. The space $\mathcal{U}$ is the eigenspace spanned by the eigenvectors associated with unstable Lyapunov exponents. These eigenvectors constitute a basis $\Phi_{\mathcal{U}}$ of $\mathcal{U}$, with $\Phi_{\mathcal{U}}(\theta) \subset \Phi(\theta)$. This implies that there exists a complementary subspace $\mathcal{S}$, spanned by $\Phi_{\mathcal{S}}$, associated with stable Lyapunov exponents i.e. $\sigma_{\mathcal{S}}(A) \equiv\{\lambda \in \mathbb{C} \mid \operatorname{Re}(\lambda)<0\}$. We emphasize that this decomposition is analogous to the usual decomposition of phase space into stable, center and unstable subspaces. Here however, for clarity of notation, we label $\mathcal{U}$ the subspace spanned by the center eigenmodes i.e. for which the eigenvalue has a zero real part, which is the usual center subspace (only the label differs).

According to the decomposition of phase space, one can project, or express, the state vector $z_{t}(\theta)$ governed by Eq. (3) with respect to the unstable basis $\Phi_{\mathcal{U}}(\theta)$,

$$
U_{t}(\theta)=P\left(z_{t}\right) \equiv \Phi_{\mathcal{U}}(\theta)\left(\Psi_{\mathcal{U}}(\theta), z_{t}(\theta)\right)=\Phi_{\mathcal{U}}(\theta)(u(t), \varepsilon)^{T}
$$

where $(u(t), \varepsilon)^{T}=\left(\Psi_{\mathcal{U}}(\theta), z_{t}(\theta)\right)$ is a vector containing the expansion amplitudes of $z_{t}(\theta)$ with respect to the unstable eigenbasis $\Phi_{\mathcal{U}}(\theta)$ and $P$ is the projector onto the subspace $\mathcal{U}$. Accordingly, the state vector $z_{t}(\theta)$ may be separated into two disjoint elements, its stable and unstable components living in $\mathcal{S}$ and $\mathcal{U}$, respectively. Consequently we can write

$$
z_{t}(\theta)=P\left(z_{t}\right)+(\mathbb{I}-P)\left(z_{t}\right)=\Phi_{\mathcal{U}}(\theta)(u(t), \varepsilon)^{T}+s_{t}(\theta)
$$

Applying the projector to the state vector of Eq. (3) yields the dynamics of the unstable modes of Eq.(2)

$$
\begin{array}{ccc}
\mathcal{U} \ni \frac{d u(t)}{d t} & =\Lambda_{\mathcal{U}} u(t)+\Psi_{\mathcal{U}}(0) F\left[\Phi_{\mathcal{U}}(\theta) u(t)+s_{t}(\theta)\right] \\
\mathcal{U} \ni \frac{d \varepsilon}{d t} & = & 0 .
\end{array}
$$

Similarly, applying the operator $\mathbb{I}-P=\mathbb{I}-\Phi_{\mathcal{U}}(\theta)\left(\Psi_{\mathcal{U}}(\theta)\right.$, · ) to Eq. (3) we obtain the complementary dynamics of the stable modes in $\mathcal{S}$

$$
\mathcal{S} \ni \frac{d}{d t} s_{t}(\theta)=\mathcal{A}\left(s_{t}(\theta)\right)+\left[X_{o}-\Phi_{\mathcal{U}}(\theta) \Psi_{\mathcal{U}}(0)\right] F\left[\Phi_{\mathcal{U}}(\theta) u(t)+s_{t}\right]
$$

\section{The autonomous center manifold reduction}

Bifurcations are characterized by so-called unstable Lyapunov exponents, or equivalently by a non-empty unstable eigenspace for non-hyperbolic fixed-points. The precise point in parameter space where $\sigma_{\mathcal{U}}(\mathcal{A}) \neq \emptyset$ is called the instability threshold, and can be quantitatively described by $\max \{\operatorname{Re}(\lambda)=0 \mid \lambda \in \sigma(\mathcal{A})\}$, which occurs in our formulation for $\epsilon=0$. In the neighborhood of a critical point, the center manifold theorem specifies that the stable modes in $\mathcal{S}$ are slaved to the dynamics of the unstable modes in $\mathcal{U}[31,35]$. Then

$$
s_{t}(\theta)=h(\theta, u(t), \varepsilon)
$$

holds true in $\mathcal{S}$. Consequently, the unstable modes are further described by the order parameter equation (OPE) 


$$
\frac{d u}{d t}=\Lambda_{\mathcal{U}} u+\Psi_{\mathcal{U}}(0) F\left[\Phi_{\mathcal{U}}(\theta) u+h(\theta, u)\right],
$$

where, within the non-linear function $F[v]$, the term $v=\Phi_{\mathcal{U}}(\theta) u+h(\theta, u)$ takes the forms $\Phi_{\mathcal{U}}(0) u+h(0, u)$ and $\Phi_{\mathcal{U}}(-\tau) u+h(-\tau, u)$, according to the timing of the non-linearities of $F$. In the vicinity of an instability and given that the functional $h(u)$ is known, the system in Eq. 10 captures the dynamics of Eq.(2) entirely. Delayed components are not present anymore, and the dimensionality of this representation is finite, making the OPE very useful in the treatment of non-linear DDEs. Most importantly, it is possible to reconstruct the flow $x(t)$ of the original delayed system of Eq.(2) solely from the unstable modes by

$$
x^{r}(t)=U_{t}(0)+s_{t}(0)=\Phi_{\mathcal{U}}(0) u(t)+h(0, u(t), \varepsilon),
$$

where $x^{r}(t)=x(t)$. Although the center manifold theorem ensures that the functional $h(\theta, u(t), \varepsilon)$ exists, it is typically not unique, and challenging to compute explicitly $[22,32,33,36]$. An approximation of $h$ introduces an error in the reconstruction leading to various degrees of discrepancy.

Nevertheless, if one combines Eq. (9) and (10), the center manifold has to satisfy the implicit relationship

$$
\begin{gathered}
D_{u} h(\theta, u, \varepsilon)\left[\Lambda u+\Psi_{\mathcal{U}}(0) F[\Phi u+h(u)]=\mathcal{A}(h(u))\right. \\
+\left[X_{o}-\Phi_{\mathcal{U}}(\theta) \Psi_{\mathcal{U}}(0)\right] F\left[\Phi_{\mathcal{U}} u+h(u),\right]
\end{gathered}
$$

where the operator $D_{u}$ denotes the partial derivative with respect to $u$. Equation (12) contains elements of both $\mathcal{S}$ and $\mathcal{U}$. However, the center manifold $h$ is normal to the space of unstable components i.e. $P(h \in \mathcal{S})=0$, a criteria we refer to as the normalization condition. This criteria, used in conjunction with Eq. (12), yields a solution $h$ which evolves in the stable subspace $\mathcal{S}$.

\section{Analysis of non-autonomous DDEs}

Now consider the non-autonomous DDE

$$
\dot{x}(t)=f(x(t), x(t-\tau), t)=L(\{x(t), x(t-\tau)\})+F(\{x(t), x(t-\tau)\})+I(t),
$$

with the external input $I(t)$.

If we consider the fixed point of the autonomous system (2) for the analysis of Eq. (13), identifying the spectrum and subspace eigenbases from the autonomous system, we may project the dynamics of the non-autonomous system in Eq. (13) onto the stable and unstable subspaces of the autonomous system with the projector $P$, and subsequently obtain the non-autonomous version of Eq. (7) and (8), namely

$$
\begin{gathered}
\mathcal{U} \ni \frac{d u(t)}{d t}=\Lambda_{\mathcal{U}} u(t)+\Psi_{\mathcal{U}}(0) F\left(\Phi_{\mathcal{U}}(\theta) u(t)+s_{t}(\theta)\right)+\Psi_{\mathcal{U}}(0) I(t) \\
\mathcal{S} \ni \frac{d}{d t} s_{t}(\theta)=\mathcal{A}_{t}(\theta)+\left(X_{o}-\Phi_{\mathcal{U}}(\theta) \Psi_{\mathcal{U}}(0)\right)\left[F\left(\Phi_{\mathcal{U}}(\theta) u(t)+s_{t}(\theta)\right)+I(t)\right]
\end{gathered}
$$


This result has been demonstrated formally for the case of a linear non-autonomous delayed system [30]. Hence the approximation is reasonable since the amplitude of both the stable and unstable modes in the vicinity of an stability threshold can be taken arbitrarily small by adjusting the control parameter, rendering the non-linear component $F$ small as well.

Equation (13) is a non-linear and non-autonomous DDE and it is a challenging problem to find conditions for its stability. Close to a bifurcation point, the analysis of such DDEs has attracted increasing attention in the last years, e.g. considering more general [30, 37], deterministic [38, 39] or stochastic forces [40, 41]. In [24], a center manifold theorem for weak solutions of non-autonomous abstract ODEs on infinite-dimensional spaces is proven given that certain spectral gap conditions are satisfied. For instance, one condition limits eigenvalues on the imaginary axis to a finite set while all other eigenvalues are bounded away from the imaginary axis. Equation (13) can be written in this setting where the external stimulation represents a non-autonomous perturbation of Eq. (3). In addition it is well-known that the autonomous operator $\mathcal{A}$ satisfies the aforementioned spectral gap conditions. As such, a time-dependent center manifold approach has been used successfully in non-delayed systems for quasi-periodic inputs [28]. Moreover the existence of time-dependent center manifolds has also been established for stochastic driving, but without delay [25]. Hence, we expect that a non-autonomous center manifold exists for Eq. (13).

As a first approximation to the delayed case, one could assume that the center manifold $h$ has no explicit dependence on time as in Eq. (9). Then the resulting calculations would yield an inconsistent problem, where there is no time-dependent component in $h$ to account for the effect of the input $I(t)$ on the non-linearities of the original DDE. The resulting reconstructed flow as per Eq. (11) would show significant deviation from the original system in Eq. (2). Thus, the present work adapts the autonomous center manifold reduction scheme to the non-autonomous case by adding an explicit time-dependence to the center manifold by means of an additive perturbation.

According to this rationale we thus choose

$$
s_{t}(\theta)=h(\theta, u(t), \varepsilon, t)
$$

for which the manifold $h(\theta, u, \varepsilon, t)$ now satisfies

$$
\begin{gathered}
D_{u} h(\theta, u, \varepsilon, t)\left[\Lambda u+\Psi_{\mathcal{U}}(0) F\left[\Phi_{\mathcal{U}} u(t)+h(u, \varepsilon, t)\right]+\Psi_{\mathcal{U}}(0) I(t)\right]+\frac{\partial h(\theta, u, \varepsilon, t)}{\partial t} \\
=\mathcal{A}(h(u, \varepsilon, t))+\left(X_{o}-\Phi_{\mathcal{U}}(\theta) \Psi_{\mathcal{U}}(0)\right)\left(F\left[\Phi_{\mathcal{U}} u(t)+h(u, \varepsilon, t)\right]+I(t)\right) .
\end{gathered}
$$

where again $h \in \mathcal{S}$.

In order to find an accurate solution to Eq. (16), it is crucial to consider the two different characteristic time scales introduced by the stable (fast) and unstable (slow) modes in the phase space decomposition. Let $\tau_{\mathcal{U}}$ and $\tau_{\mathcal{S}}$ be the time scales of the unstable and stable modes, respectively. In the mindset of the center manifold theorem and following the hypothesis of the existence of non-hyperbolic fixed points, we assume that the forcing term is fast, so that $I(t)=I\left(\tau_{f} t^{\prime}\right)$, where $\tau_{f} \leq \tau_{\mathcal{S}}<<\tau_{\mathcal{U}}$. More formally, $\tau_{f}, \tau_{\mathcal{S}} \sim \varepsilon^{m}, \tau_{\mathcal{U}} \sim \varepsilon^{n}$ with $m \geq n$. In addition, it is reasonable to assume small amplitudes close to the bifurcation threshold yielding $u \sim \varepsilon^{n}$. As an ansatz, we add a time-dependent correction $h_{t}$ to the expansion used in the autonomous case, such that the time-dependence in the center manifold takes the form of a fast and small additive perturbation

$$
h\left(\theta, u, t, t^{\prime}\right)=h_{n}(\theta, \varepsilon, u)+h_{t}\left(\theta, \tau_{f} t^{\prime}\right)+\mathcal{O}(m>n),
$$

where $\mathcal{O}(m)$ denotes terms of order of magnitude $m$ in $u, \varepsilon$ and the time-dependent contributions. The ansatz in Eq. (17) assumes that the autonomous center manifold $h_{m}$ of order $m$ and the correction term $h_{t}$ have similar orders of magnitude: we assume $u \sim \varepsilon$ and $h_{n} \sim \mathcal{O}(n \geq 2), h_{t} \sim \mathcal{O}(2), I(t) \sim \mathcal{O}(2)$, i.e. the ansatz implies time-dependent corrections and inputs that are small compared to the amplitude of the unstable modes. Furthermore, such an ansatz is analogous to the one used in [28] for forced center manifolds in the context of ODEs, which proved to accurately reproduce the dynamics for various types of inputs. It assumes that the center manifold has a separable form in time $t$ and modes $u$, which greatly simplifies the resolution of Eq. (16). Indeed, the substitution of (17) in Eq. (16) up to quadratic order leads both to the evolution equations of the autonomous problem and the non-autonomous evolution 
equation on a faster time scale. The resulting evolution of $h_{t}$ is decoupled from the autonomous contribution $h_{n}(\theta, u)$ and obeys

$$
\frac{\partial h_{t}(\theta, t)}{\partial t}=\mathcal{A}(\theta)\left(h_{t}\right)+\left[X_{o}(\theta)-\Phi_{\mathcal{U}}(\theta) \Psi_{\mathcal{U}}(0)\right] I(t)
$$

Equation (18) is a linear first order non-homogeneous partial differential equation in $h_{t}$. In order to be solved, recall that $h_{t}$ evolves in the stable subspace, i.e. $P\left(h_{t}\right)=0$. Hence it is reasonable to choose the ansatz

$$
h_{t}(\theta, t)=(\mathbb{I}-P) H(t+\theta)
$$

where $H \in \mathcal{C}$ is a continuous scalar function.

To derive the evolution equation for $H(t+\theta)$, at first let us consider the case $-\tau \leq \theta<0$. Then inserting Eq. (19) into Eq. (18) yields

$$
\frac{\partial}{\partial t} P(H)=\frac{\partial}{\partial \theta} P(H)+\Phi_{\mathcal{U}}(\theta) \Psi_{\mathcal{U}}(0) I(t)
$$

In the following, we assume bifurcations of co-dimension 1. Since $(\partial / \partial \theta) P(H)=\left(\partial \Phi_{\mathcal{U}} / \partial \theta\right)\left(\Psi_{\mathcal{U}}, H\right)=0$ and

$$
P\left(\frac{\partial H}{\partial t}\right)=\Phi_{\mathcal{U}}(\theta) \Psi_{\mathcal{U}}(0)\left(\frac{d H}{d t}-L[H]\right)
$$

the resulting evolution equation reads

$$
\frac{\partial H(t+\theta)}{\partial t}=L[H]+I(t) \quad, \quad-\tau \leq \theta<0 .
$$

Since the right hand side of (21) does not depend on $\theta, H(t+\theta)=H(t),-\tau \leq \theta<0$.

Similarly, for $\theta=0$ we find

$$
\left(1-\Phi_{\mathcal{U}}(0) \Psi_{\mathcal{U}}(0)\right) \frac{d H(t)}{d t}=\left(1-\Phi_{\mathcal{U}}(0) \Psi_{\mathcal{U}}(0)\right) L[H]-L[P(H)]+\left(1-\Phi_{\mathcal{U}}(0) \Psi_{\mathcal{U}}(0)\right) I(t)
$$

Since

$$
\begin{aligned}
L[P(H)] & =\left(\Psi_{\mathcal{U}}, H\right) \int_{-\tau}^{0} \Phi_{\mathcal{U}}(\theta)[d \eta(\theta)] \\
& =\left(\Psi_{\mathcal{U}}, H\right) L\left[\Phi_{\mathcal{U}}\right] \\
& =0
\end{aligned}
$$

One finds that Eq. (22) is identical to Eq. (21). Hence Eq. (21) determines $H(t+\theta)$ and $h_{t}$ via Eq. (19).

Although Eq. (21) is still non-autonomous, it is linear and may be solved by various techniques dependent on the specific problem at hand and on the forcing term $I(t)[30,42,43]$. In addition, it is worth mentioning that the maximum eigenvalue of $L[H]$ vanishes for co-dimension 1 bifurcations. This may render the solution $H(t)$ of Eq. (21) non-stationary, e.g. in the presence of Brownian noisy inputs as shown below in Section III B 1.

Summarizing this section, we propose an approach built on the hypothesis that both non-autonomous and delayed cases can be analyzed, in a lowest order approximation, by a fast and small time-dependent correction of the autonomous center manifold of nonlinear systems. The separation of the mode-dependent parts $h_{n}$ and the time-dependent part $h_{t}$, combined with the time-scale separation asumption allows to compute higher order terms in the modes expansion, while keeping the time-dependent component to second order. Higher degrees of accuracy could be achieved by computing higher order terms in both modes and time-dependent components of $h(\theta, u, \varepsilon, t)$, in Eq.(17) for instance. In addition, the method allows to reduce dramatically the dimensionality of the delayed system by deriving a low-dimensional non-delayed system, the so-called order parameter equation.

The subsequent section illustrates the method and investigates its validity by the application to a specific delayed problem. 


\section{APPLICATION TO THE ASYMMETRICAL TRANSCRITICAL BIFURCATION}

Consider the following scalar delayed system with the quadratic normal form

$$
\frac{d x(t)}{d t}=-\left[x(t)-R_{1} x(t-\tau)\right]-R_{2} x^{2}(t-\tau)+I(t),
$$

Such normal form structures embody a vast array of systems, whose non-linearities have been expanded near a given fixed point and where quadratic components dominate. Numerous examples exist where such a situation arise, especially in applications where the dynamics is subject to a delayed feedback component expressing a sigmoidal behavior. It has indeed been shown that the center manifold for a system with quadratic nonlinearity affects the deterministic dynamics [35]. The treatment of the cubic case is analogous to the one we perform here, and is presented elsewhere.

Introducing the new constant parameter $\varepsilon \equiv R_{1}+1$, Eq. (23) may be augmented to

$$
\begin{aligned}
\frac{d x(t)}{d t} & =-x(t)+x(t-\tau)-\varepsilon x(t-\tau)-R_{2} x^{2}(t-\tau)+I(t) \\
\frac{d \varepsilon}{d t} & =0 .
\end{aligned}
$$

As we will see below, $\varepsilon$ serves as the control parameter of the system. Also, note that the augmented system treats $\varepsilon$ as a dynamical variable. This artificially extended formulation has the advantage of having a vanishing eigenvalue of the linear system for all values of the control parameter, which permits the application of the center manifold reduction even away from the bifurcation.

Introducing the vector function $z_{t}(\theta)=(x(t+\theta), \varepsilon)^{T} \equiv\left(z_{t}(\theta)_{1}, z_{t}(\theta)_{2}\right)^{T}$, the corresponding operators in the extended space $\mathcal{C}$ read

$$
\begin{aligned}
& L\left[z_{t}\right]=\left(\begin{array}{c}
-z_{t}(0)_{1}+z_{t}(-\tau)_{1} \\
0
\end{array}\right)=\int_{-\tau}^{0} d \eta[\theta] z_{t}(\theta) \\
& F\left[z_{t}\right]=-\left(\begin{array}{c}
\varepsilon z_{t}(-\tau)_{1}+R_{2} z_{t}^{2}(-\tau)_{1} \\
0
\end{array}\right)
\end{aligned}
$$

with

$$
d \eta[\theta]=\left(\begin{array}{cc}
-\delta(\theta)+\delta(\theta+\tau) & 0 \\
0 & 0
\end{array}\right) d \theta .
$$

At first, we will investigate the problem without driving, i.e. $I(t)=0$, to illustrate the procedure of delayed center manifold reduction. Then the following paragraphs show a detailed derivation of the time-dependent correction to the autonomous center manifold for $I(t) \neq 0$.

\section{A. Autonomous Case: $I(t)=0$}

Fixed points of Eq.(23) for $I(t)=0$ are given by

$$
x_{1,2}^{o}=0,-\frac{\left(1+R_{1}\right)}{R_{2}} .
$$

A transcritical bifurcation occurs at $R_{1}=-1$, for which the stable branch $x_{s}^{o}\left(R_{1}\right)=-\left(1+R_{1}\right) R_{2}^{-1}$ and unstable branch $x_{u}^{o}\left(R_{1}\right)=0$ collide and exchange stability. This is further made apparent by investigating the spectrum $\sigma(L)$ of the linearized problem $\dot{x}(t)=L(x(t), x(t-\tau))=-x(t)-R_{1} x(t-\tau)$ about $x_{1}^{o}=0$. The corresponding characteristic polynomial is

$$
\Delta\left(\lambda, R_{1}\right) \equiv \lambda+1+R_{1} e^{-\lambda \tau}=0
$$

with the roots $\lambda \in \mathbb{C}$. Some solutions of the characteristic equation are plotted in Fig.1.

We notice that $\mathbb{R} \ni \lambda=0$ for $R_{1}=-1$. In order to capture the dynamics in a neighbourhood of the instability, we might consider the augmented system in Eq. (24). Its linear part reads 


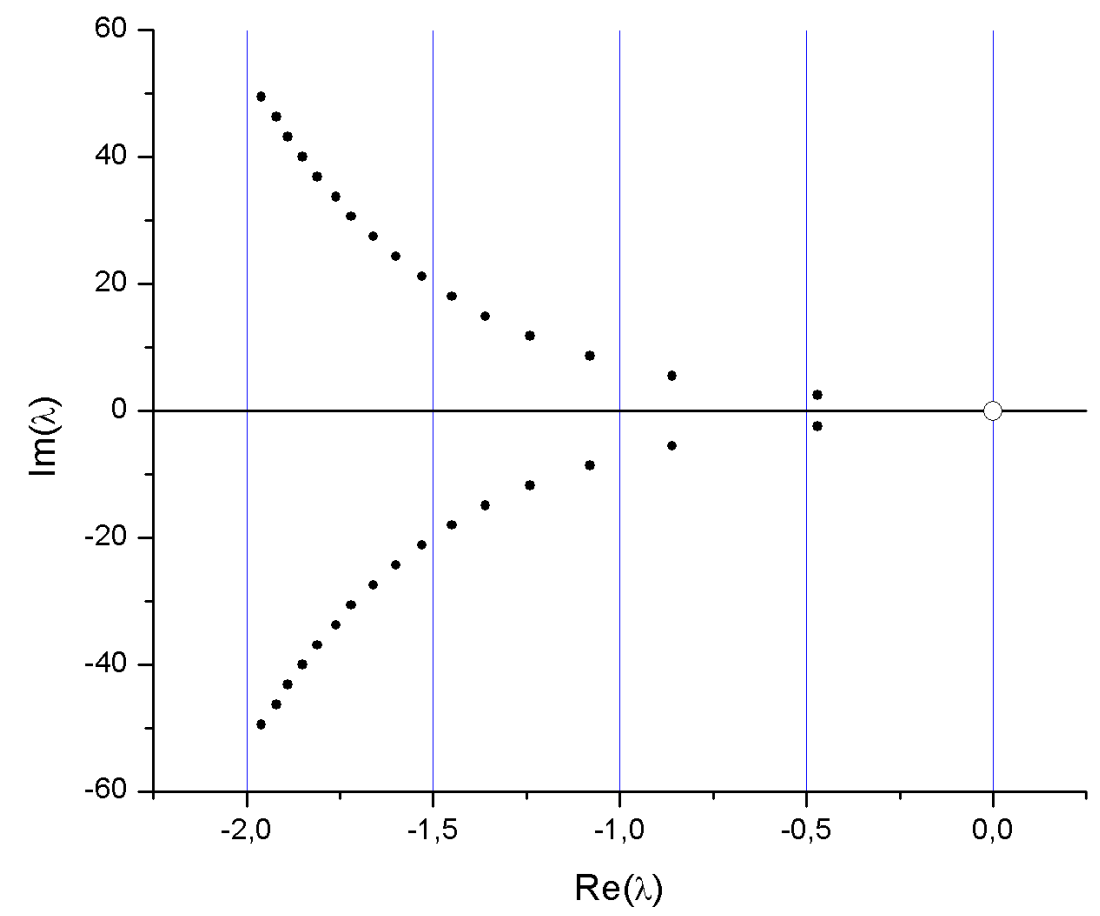

FIG. 1: Lyapunov exponents $\lambda$ of Eq.(23) at the trivial equilibrium for $R_{1}=-1$. Stable exponents are shown in black, while the only unstable exponent is shown as an open circle. Here, $\tau=2.0$.

$$
\begin{aligned}
\frac{d x(t)}{d t} & =-x(t)+x(t-\tau) \\
\frac{d \varepsilon}{d t} & =0
\end{aligned}
$$

whose characteristic polynomial is $\lambda \Delta(\lambda,-1)=0$. We observe that the Lyapunov exponents of the augmented system are now independent from the order parameter $\varepsilon$ by construction. The corresponding orthonormal eigenbasis of the unstable subspace, i.e. for $\lambda=0$, reads

$$
\Phi_{\mathcal{U}}(\theta)=\left(\begin{array}{ll}
1 & 0 \\
0 & 1
\end{array}\right)=\mathbb{I}_{2}
$$

The biorthonormal adjoint basis can be computed using Eq.(5) and $\Psi_{\mathcal{U}}=\left(\Phi_{\mathcal{U}}^{T}, \Phi_{\mathcal{U}}\right)^{-1} \Phi_{\mathcal{U}}^{T}$ to yield

$$
\Psi_{\mathcal{U}}(\theta)=\left(\begin{array}{cc}
(1+\tau)^{-1} & 0 \\
0 & 1
\end{array}\right)
$$

where now $\left(\Phi_{\mathcal{U}}(\theta), \Psi_{\mathcal{U}}(\theta)\right)=\mathbb{I}_{2}$. In addition, the projector $P(\cdot)=\Phi_{\mathcal{U}}(\theta)\left(\Psi_{\mathcal{U}}(\theta), \cdot\right)$ onto the unstable subspace is

$$
P(\phi(\Theta))=(1+\tau)^{-1}\left(\phi(0)+\int_{-\tau}^{0} \phi(s) d s\right)
$$

A projection of Eq. (24) onto $\mathcal{U}$ and the complementary space $\mathcal{S}$ yields 


$$
\begin{aligned}
\frac{d u(t)}{d t} & =(1+\tau)^{-1} F\left[u+s_{t}\right] \\
\frac{d \varepsilon(t)}{d t} & =0 \\
\frac{d}{d t} s_{t}(\theta) & =\mathcal{A}\left(s_{t}\right)+\left(X_{o}-\frac{1}{1+\tau}\right) F\left[u+s_{t}\right]
\end{aligned}
$$

where the linear operator $\mathcal{A}$ contains the linear functional $L\left[s_{t}\right]$ and the nonlinear part is $F\left[u+s_{t}\right]=-\varepsilon x(t-\tau)-$ $R_{2} x^{2}(t-\tau)$. To obtain $F$ as a function of the projection variables $u$ and $s_{t}(\theta)$, respectively, we recall the definition of $z_{t}(\theta)$ and find $x(t-\tau)=\Phi(-\tau) u(t)+s_{t}(-\tau)=u(t)+s_{t}(-\tau)$. Consequently the linear and nonlinear part read

$$
\begin{aligned}
L\left[s_{t}\right] & =-s_{t}(0)+s_{t}(-\tau), \\
F\left[u+s_{t}\right] & =-\varepsilon\left(u(t)+s_{t}(-\tau)\right)-R_{2}\left(u(t)+s_{t}(-\tau)\right)^{2} .
\end{aligned}
$$

We may now apply the center manifold theorem. The fast stable modes are slaved to the slow unstable modes, i.e. $s_{t}(\theta)=h(\theta, u(t), \varepsilon)$, where the functional $h$ obeys

$$
\frac{1}{1+\tau} \frac{\partial h(\theta, u, \varepsilon)}{\partial u} F(u(t)+h(\theta, u))=\mathcal{A}(h(\theta, u, \varepsilon))+\left(X_{o}-\frac{1}{1+\tau}\right) F(u(t)+h(\theta, u, \varepsilon)) .
$$

As mentioned previously, Eq. (34) can be solved by introducing an order of occuring terms and the subsequent solution of each order separately.

\section{Quadratic terms}

First, let us find the manifold $h(\theta, u, \varepsilon)$ up to second order in $(u, \varepsilon)$ with the quadratic ansatz

$$
h(\theta, u, \varepsilon) \approx h_{2}(\theta, u, \varepsilon)=a(\theta) \varepsilon^{2}+b(\theta) \varepsilon u+c(\theta) u^{2}+\mathcal{O}(3 ; \varepsilon, u) .
$$

The coefficients $a(\theta), b(\theta), c(\theta) \in \mathbb{R}$ are arbitrary functions. For $-\tau \leq \theta<0$ Eq. (34) reads

$$
\frac{1}{1+\tau} \frac{\partial h_{2}(\theta, u, \varepsilon)}{\partial u} F[u+h(u, \varepsilon)]=\frac{\partial h_{2}(\theta, u, \varepsilon)}{\partial \theta}-\frac{1}{1+\tau} F[u+h(u, \varepsilon)] .
$$

Since $F$ and $h_{2}$ are $\mathcal{O}(2)$, Eq. (36) becomes

$$
\frac{\partial h_{2}(\theta, u, \varepsilon)}{\partial \theta}=\frac{1}{1+\tau} F[u(t)]
$$

Substituting the polynomial ansatz of Eq. (35) in the previous equation gives, to second order,

$$
a^{\prime}(\theta) \varepsilon^{2}+b^{\prime}(\theta) \varepsilon u+c^{\prime}(\theta) u^{2}=\frac{1}{1+\tau}\left(-\varepsilon u-R_{2} u^{2}\right) .
$$

Collecting the orders of $(u, \varepsilon)$, the coefficients of the center manifold are governed by a decoupled system of ODEs with the solutions

$$
\begin{aligned}
& a(\theta)=C_{1} \\
& b(\theta)=-(1+\tau)^{-1} \theta+C_{2} \\
& c(\theta)=-(1+\tau)^{-1} R_{2} \theta+C_{3}
\end{aligned}
$$


and constants $C_{1}, C_{2}, C_{3} \in \mathbb{R}$. Reworking the problem for $\theta=0$ does not allow to determine these constants. We recall the normalization condition $P\left(h_{2}\right)=0$, where $P$ is defined in Eq. (30). Then utilizing Eq. (38) in $P\left(h_{2}\right)=0$ yields $C_{1}=0, C_{2}=\tau^{2} / 2(\tau+1)^{2}$ and $C_{3}=R_{2} \tau^{2} / 2(\tau+1)^{2}$. Hence the lowest order of the autonomous center manifold of Eq. (23) is

$$
h_{2}(\theta, u, \varepsilon)=\underbrace{\left(\frac{\theta}{\tau+1}+\frac{\tau^{2}}{2(\tau+1)^{2}}\right)}_{b(\theta)} u \varepsilon-\underbrace{\left(\frac{\theta R_{2}}{\tau+1}+\frac{R_{2} \tau^{2}}{2(\tau+1)^{2}}\right)}_{c(\theta)} u^{2}
$$

\section{Cubic terms}

For the cubic components, the very same procedure applies and so we simply state the result. The cubic component of the center manifold is

$$
\begin{gathered}
h_{3}(\theta, u, \varepsilon)=\left(\frac{1}{2} \frac{\theta^{2}}{(1+\tau)^{2}}-\theta \frac{\tau}{(1+\tau)^{2}}-\frac{2 \tau^{3}}{3(\tau+1)^{3}}\right) u \varepsilon^{2} \\
+\left(R_{2} \frac{\theta^{2}}{(1+\tau)^{2}}-\theta \frac{2 R_{2} \tau}{(1+\tau)^{2}}-\frac{4 R_{2} \tau^{3}}{3(\tau+1)^{3}}\right) \varepsilon u^{2} \\
+\left(R_{2}^{2} \frac{\theta^{2}}{(1+\tau)^{2}}-\theta \frac{2 R_{2}^{2} \tau}{(1+\tau)^{2}}-\frac{4 R_{2}^{2} \tau^{3}}{3(\tau+1)^{3}}\right) u^{3} .
\end{gathered}
$$

Combining the expression for the center manifold obtained at the second and third order, the order parameter equation for the autonomous case reads

$$
\frac{d u(t)}{d t}=(1+\tau)^{-1}\left[-\varepsilon(u+h(-\tau, u))-R_{2}(u+h(-\tau, u))^{2}\right]
$$

with $h=h_{2}+h_{3}$. Figure 2 shows an example of the effect of higher order terms calculations on the level of accuracy achieved by the order parameter equation in the description of the original system in Eq. (23). The figure compares the solution of the original system of Eq. $(23)$, to the reconstructed flow $x_{r}(t)=\Phi_{\mathcal{U}}(0) u(t)+h(0, u)$ at diffferent orders of expansion of the center manifold. As expected, the best approximation is by the center manifold of cubic order, i.e. $h=h_{2}+h_{3}$, and decreases for the lower orders $h=h_{2}$, and more so for $h=0$.

\section{B. Non-autonomous case: $I(t) \neq 0$}

Let us now investigate Eq. (23) with $I(t) \neq 0$. As discussed previously, the analysis of the linear problem yields the same dynamics as in the autonomous case, since the external input is treated as a non-linearity. Following the construction of the eigenbases, we project Eq.(23) onto $\Phi_{\mathcal{U}}$ and the complementary basis $\Phi_{\mathcal{S}}$, and obtain

$$
\begin{aligned}
\frac{d u(t)}{d t} & =\frac{1}{1+\tau} F\left[u(t)+s_{t}\right]+\frac{1}{1+\tau} I(t) \\
\frac{d \varepsilon(t)}{d t} & =0 \\
\frac{d}{d t} s_{t}(\theta) & =\mathcal{A}\left(s_{t}\right)+\left(X_{o}-\frac{1}{1+\tau}\right)\left(F\left[u(t)+s_{t}\right]+I(t)\right) .
\end{aligned}
$$

Now applying the center manifold theorem implies that the functional $h$ depends on time explicitely. We obtain

$$
\begin{aligned}
& \frac{1}{1+\tau} \frac{\partial h(\theta, u, t)}{\partial u}(F[u(t)+h(u, t)]+I(t))+\frac{\partial h(\theta, u, t)}{\partial t} \\
= & \mathcal{A}(h(u, t))+\left(X_{o}-\frac{1}{1+\tau}\right)(F[u(t)+h(u, t)]+I(t)) .
\end{aligned}
$$




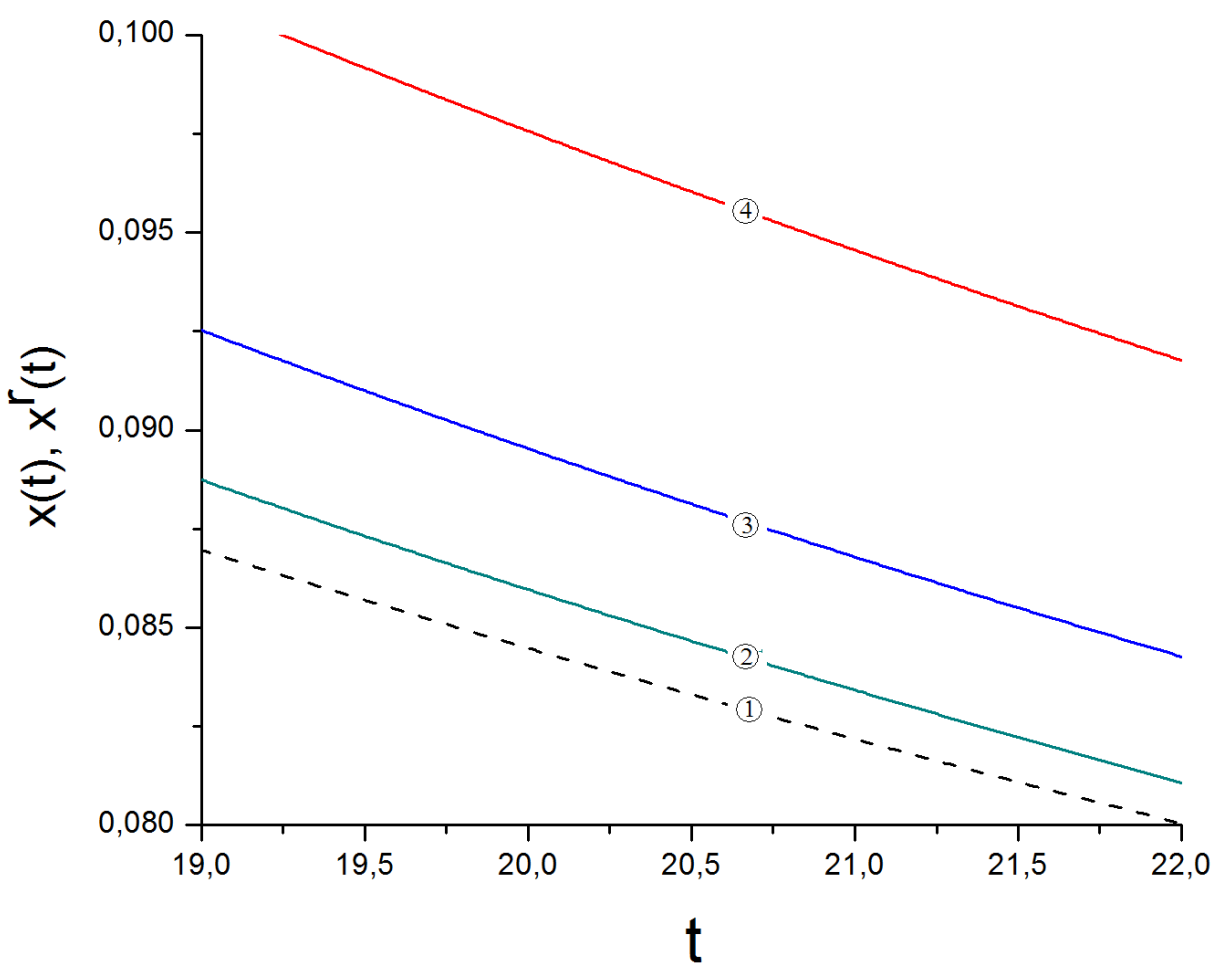

FIG. 2: Comparison of the numerically reconstructed flow $x^{r}(t)$ defined by Eq.(11) with the original flow of Eq.(23) for I $(t)=0$ based on the calculations performed in this section. For a specific initial condition, the solution of Eq. (23) approaches the fixed point $x_{o}^{1}=0$. The reconstructed flow is shown with different accuracy levels achieved using higher order terms in the center manifold formulation. To approach the original flow (dashed curve labelled 1), the order parameter equation (Eq.(41)) uses cubic (2) and quadratic (3) order expansions for the center manifold. The trivial ansatz $h=0$ (4) is also shown. Parameters are $\tau=2.0, \varepsilon=-0.05, R_{2}=1.5$. Initial conditions were chosen to exhibit an monotonic decay towards the equilibrium and were the same for all simulations over the interval $-\tau<t_{o}<0$, so that $x\left(t_{o}\right)=x_{r}\left(t_{o}\right)=u\left(t_{o}\right)=0.3$ where we have $h\left(0, u\left(t_{o}\right)\right)=0$. An Euler integration scheme was used.

For simplicity, we do not write the dependence of $h$ on $\varepsilon$. Considering the solution $h(\theta, u, t)$ in $(42)$ yields the order parameter equation

$$
\frac{d u(t)}{d t}=\frac{1}{1+\tau}\left(-\varepsilon(u+h(-\tau, u, t))-R_{2}(u+h(-\tau, u, t))^{2}\right)+\frac{1}{1+\tau} I(t) .
$$

Solving Eq. (43) for $h(\theta, u, t)$, we take into account that the forcing term is small compared to the evolution of the unstable mode with $I(t) \sim \mathcal{O}(2)$. Implementing this time-dependence of the center manifold, we may write the center manifold in time-mode separable form

$$
h(\theta, u, \varepsilon, t)=h_{n}(\theta, u(t), \varepsilon)+h_{t}(\theta, t), .
$$

The determination of the time-dependent component $h_{t}$ is facilitated by the ansatz $h_{t}=(\mathbb{I}-P) H(t+\theta)$ which holds on the normalization criterion. According to Eq. (21), we find the evolution equation

$$
\frac{\partial h_{t}(\theta, t)}{\partial t}=\mathcal{A}(\theta)\left(h_{t}\right)+\left[X_{o}(\theta)-\frac{1}{1+\tau}\right] I(t),
$$

and the solution ansatz

$$
\begin{aligned}
h_{t} & =(\mathbb{I}-P) H(t+\theta) \\
& =H(t+\theta)-\frac{H(t)}{1+\tau}-\frac{1}{1+\tau} \int_{-\tau}^{0} H(t+s) d s,
\end{aligned}
$$


which may be simplified to

$$
\dot{H}(t+\theta)=-H(t)+H(t-\tau)+I(t)
$$

Given a specific choice for the input $I(t)$, this expression fully determines the time-dependent componnent of the center manifold.

\section{Periodic driving}

To verify these results, let us consider the periodic driving $I(t)=I_{o} \sin \left(w_{o} t\right)$ with a single angular frequency $w_{o}$. The amplitude $I_{o}$ is assumed to be small compared to the amplitude $u(t)$, and the oscillation period $2 \pi / w_{o}$ is short compared to the slow evolution of $u(t)$. The time-dependent correction $h_{t}(\theta, t)$ is a solution of Eq. (45) whenever $H$ satisfies the linear delay equation

$$
\frac{d H(t)}{d t}=-H(t)+H(t-\tau)+I_{o} \sin \left(w_{o} t\right)
$$

In the absence of an external input, the solution of $d H_{0} / d t=L\left[H_{0}\right]=-H_{0}(t)+H_{0}(t-\tau)$ is $H_{0}(t)=$ $\sum_{n} c_{n} \exp \left(\lambda_{n} t\right),-\infty<n<\infty$ with constants $c_{n}=c_{-n}^{*} \in \mathbb{C}$ and Lyapunov exponents $\lambda_{n} \in \mathbb{C}$. The eigenspectrum of $L[H]$ is depicted in Fig. 1, and we may observe that $\lambda_{0}=0, \operatorname{Re}\left(\lambda_{n \neq 0}\right)<0$. Hence $H=$ const is a stable fixed point. For $I \neq 0$, the solution of (48) reads for $t \rightarrow \infty$,

$$
H_{o}(t)=A_{o} \sin \left(w_{o} t\right)+B_{o} \cos \left(w_{o} t\right)
$$

with constants $A_{o}=A_{o}\left(I_{o}, w_{o}, \tau\right), B_{o}=B_{o}\left(I_{o}, w_{o}, \tau\right)$. Then (47) yields the time correction $h_{t}$

$$
\begin{aligned}
h_{t}(\theta, t)= & A_{o} \sin \left(w_{o}(t+\theta)\right)+B_{o} \cos \left(w_{o}(t+\theta)\right) \\
& -\left(\frac{A_{o}}{1+\tau}+\frac{B_{o}}{w_{o}(1+\tau)}\right) \sin \left(w_{o} t\right)-\left(\frac{B_{o}}{1+\tau}+\frac{A_{o}}{w_{o}(1+\tau)}\right) \cos \left(w_{o} t\right) \\
& +\frac{A_{o}}{w_{o}(1+\tau)} \cos \left(w_{o}(t-\tau)\right)+\frac{B_{o}}{w_{o}(1+\tau)} \sin \left(w_{o}(t-\tau)\right) .
\end{aligned}
$$

Combining the results from previous sections, we obtain the final expression for the non-autonomous center manifold up to the cubic order, and the resulting order parameter equation for the specific forcing considered here.

In order to evaluate our method, we numerically integrated the original system Eq.(23) and compared the resulting solution to the solution obtained according to Eq.(11). The results are presented in Fig. 3. There, we have considered the two cases with and without time-dependence of the center manifold. However, both approaches share the same autonomous component, while the time-dependent manifold obviously exhibits an additional time-dependent term. One can clearly see that the time-dependent correction of the center manifold significantly improves the correspondance between the original and reconstructed signals.

The reduction method assumes a time-scale separation of the fast external input and the slow dynamics of $u(t)$. In addition, the amplitude of $I(t)$ and the time-dependent correction term $h_{t}$ are assumed to be small compared to $u(t)$, and one can expect the error to increase as the input amplitude, $I_{o}$, increases. To illustrate how both the input oscillation frequency and the input amplitude affect the reconstruction error, we computed in Fig. 4 the time-averaged difference between original and reconstructed flows with sinusoidal driving of various amplitudes and frequencies. One can see that the inclusion of a time-dependent center manifold reduces the reconstruction error, especially for smaller frequencies. Moreover, in both cases with or without time-dependent center manifold corrections, increasing the frequency and decreasing the input amplitude reduces the error. For very small values of the frequency and/or large input amplitudes, the solutions diverge away from the equilibrium, as the input causes the flow to make large excursions out of the basin of attraction of the fixed point $x_{o}$. These conditions correspond to points in the figure which exhibit a maximal error.

The exact interval of frequencies and amplitudes for which the time-dependent center manifold calculations can be used is hard to determine. The rule of thumb is that small input amplitudes and fast oscillating inputs allow a good 


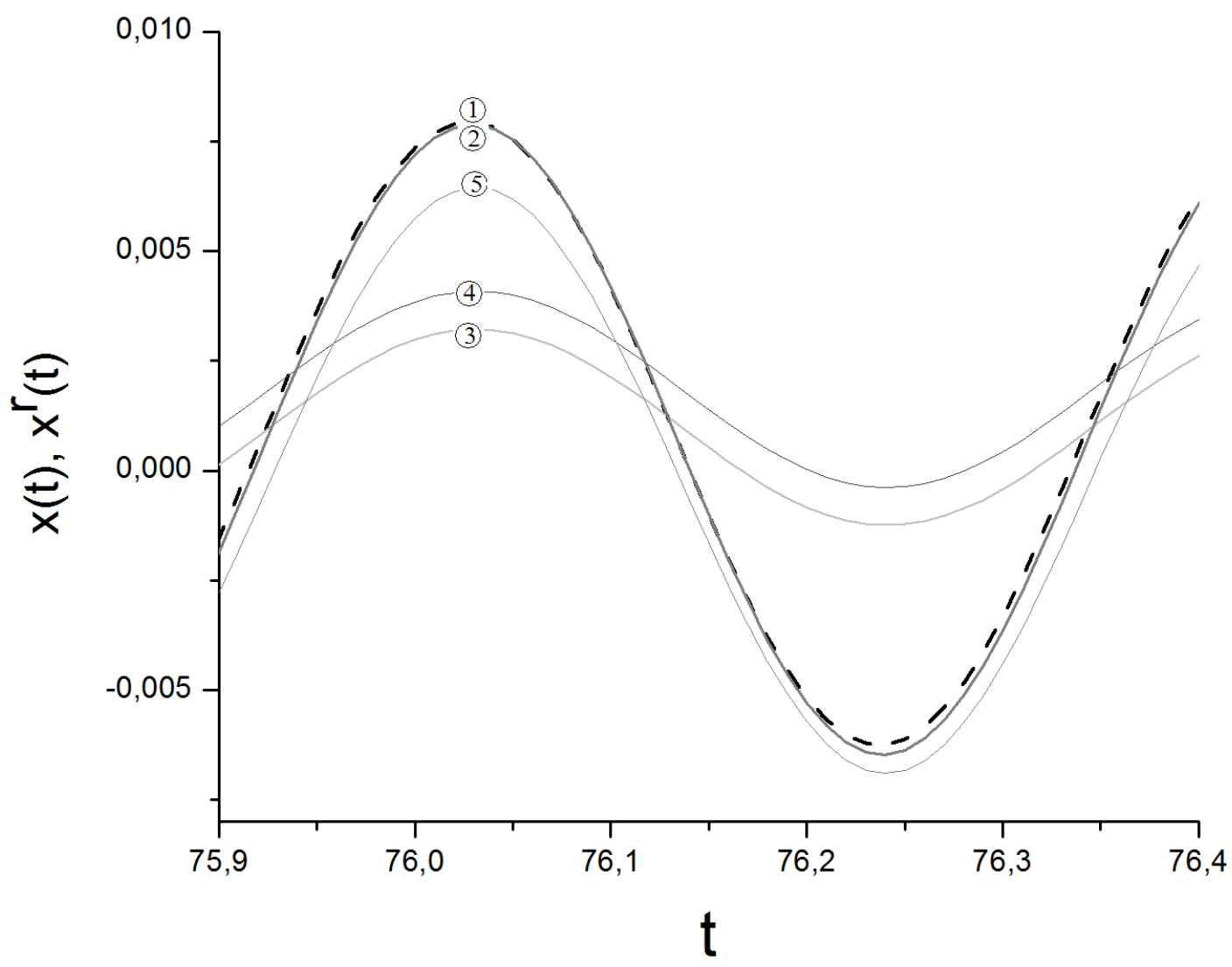

FIG. 3: Comparison of numerical simulations of the reconstructed flow $x^{r}(t)$ from Eq.(11) with the original flow of Eq.(23) with weak and fast time-periodic driving of the form $I(t)=I_{o} \sin \left(w_{o} t\right)$. The numerical solution of Eq. (23) (dashed curve labelled1) is shown along with the reconstructed flow with cubic order expansion ( $h=h_{2}+h_{3}$ in Eq. (39) and (40)) of the center manifold, and plotted with (2) and without (3) the time-dependent correction $h_{t}$ (Eq. 49). The reconstructed flow using the tivial ansatz $h=0$ is also shown (4). Further, we add the flow of Eq. (23) without any delay (i.e. for $\tau=0)(5)$, showing the difference in the system's response to forcing when no delay is present, and how this difference is accurately captured by the time-dependent center manifold approach. The time-corrected center manifold follows the solution of the DDE with improved accuracy. Parameters are $I_{o}=0.1, w_{o}=15, \tau=2.0, \varepsilon=0.15, R_{2}=1.5$. The initial conditions are chosen the same for each system i.e. $x\left(t_{o}\right)=x_{r}\left(t_{o}\right)=u\left(t_{o}\right)=0.3$ for $-\tau \leq t_{o}<0$, where we set $h\left(0, u\left(t_{o}\right)\right)=0$.

approximation. In line with the principal objective of center manifold theory, we provide here a simple method for computing a time-dependent center manifold of low order for driven delayed systems, which greatly improves the accuracy of the order parameter equation. Further, our method is based on the calculation of the center manifold of the autonomous problem, for which the literature provides numerous detailed examples on which one can add non-autonomous extensions.

\section{Driving at multiple frequencies}

As observed in the previous paragraphs, the proposed analysis assumes a short time scale of the oscillating input, such that low input frequencies yield a larger error between the original flow and the reconstructed flow than higher frequencies. However, in real applications external driving forces may not be periodic in time as assumed in the previous paragraphs. Hence the question arises whether the proposed method still applies to more general inputs. To consider this question, let us consider the more general quasi-periodic driving $I(t)$ whose time scales are assumed 

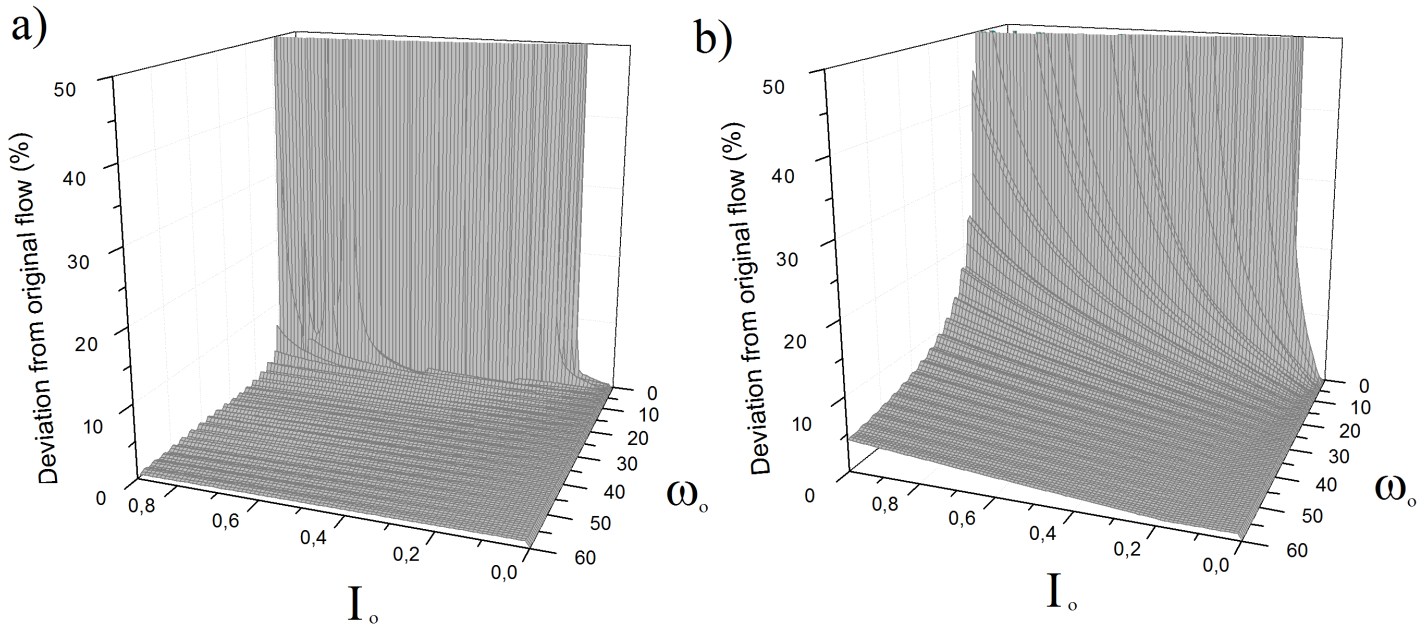

FIG. 4: Deviations between the reconstructed flow governed by Eq. (44) and the original flow of Eq. (23) near a transcritical bifurcation using both standard and time-dependent center manifold reductions with an additive forcing term $I(t)=I_{o} \sin \left(w_{o} t\right)$. As the two flows are in phase, the error is defined as the absolute difference between original and recontructed flows at input maxima divided by the response amplitude of the original system, taken at the maxima of the oscillation. Panel a) shows the error when an additive time-dependent component to the center manifold is used, as in Eq. 49. Panel b) shows the error for the same problem without any time-dependent considerations in the center manifold calculations. Parameters are $\tau=2.0, \varepsilon=-0.2$ and $R_{2}=1.5$.

short. Assuming a finite set of oscillations of number $N$, the input may be written as

$$
I(t)=\sum_{i=1}^{N} I_{o}^{i} \sin \left(w_{o}^{i} t\right) .
$$

It is obvious that the choice of the frequency distribution $\left\{I_{o}^{i}\right\}$ and especially its low-frequency part determines the fit of the reconstructed signal to the original signal. Following the previous analysis procedure, the autonomous center manifold can be approximated up to cubic order. Since the center manifold depends linearly on the external input, it is easy to check that the function $H$ introduced in Eq. (19) now simply obeys

$$
\frac{d H(t)}{d t}=-H(t)+H(t-\tau)+\sum_{i=1}^{N} I_{o}^{i} \sin \left(w_{o}^{i} t\right)
$$

similar to the single frequency case (48). By virtue of the linearity of the problem, the solution of (51) represents a linear superposition of the solutions (49) for different input strengths and frequencies. Figure 5 compares a single solution of the full model and some reconstructed solutions for a pulse-like external input, in the same spirit as in Fig. 3. We may also observe in this case how the time-dependent term $h_{t}$ improves the reconstruction very well. The reconstruction error is determined by the smallest frequencies and dominant amplitudes chosen (not shown).

\section{White noise}

The results of Fig. 3 and 5 show that the reduction method is valid for inputs composed of a single frequency and also for superimposed oscillations of different frequencies. Let us consider the general case of a superposition of infinitely many frequencies, i.e. noise which is uncorrelated in time. The input now takes the form $I(t)=\kappa \xi(t)$ where $\kappa$ denotes the noise strength and $\xi(t)$ is a random variable taken from a Gaussian distribution with $\langle\xi(t)\rangle=0$ and correlation $\langle\xi(t) \xi(T)\rangle=2 \delta(t-T)$. Here $\langle\cdots\rangle$ denotes the average over time. The input power spectrum is a constant and thus all Fourier frequencies are assumed to have identical amplitude. This input choice extends the study of the determination of delayed center manifolds to the general case of stochastic inputs.

The noise strength $\kappa$ is assumed to be small compared to the amplitude $u(t)$. Similar to the discussion of 
(a)

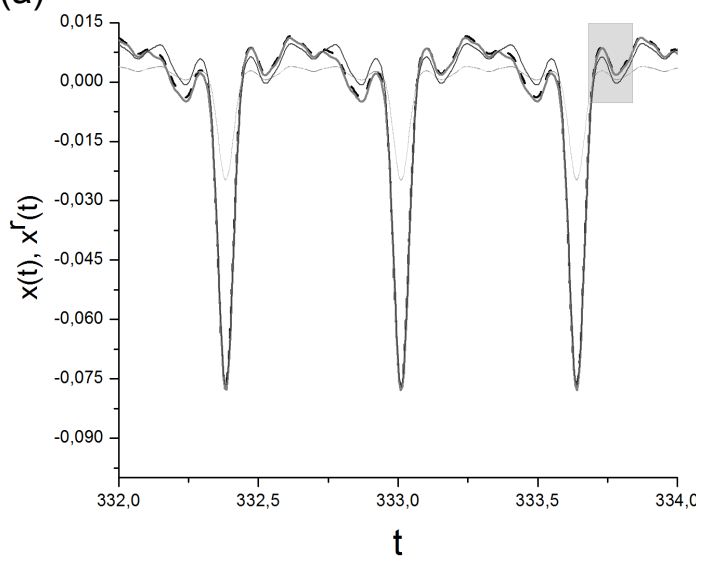

(b)

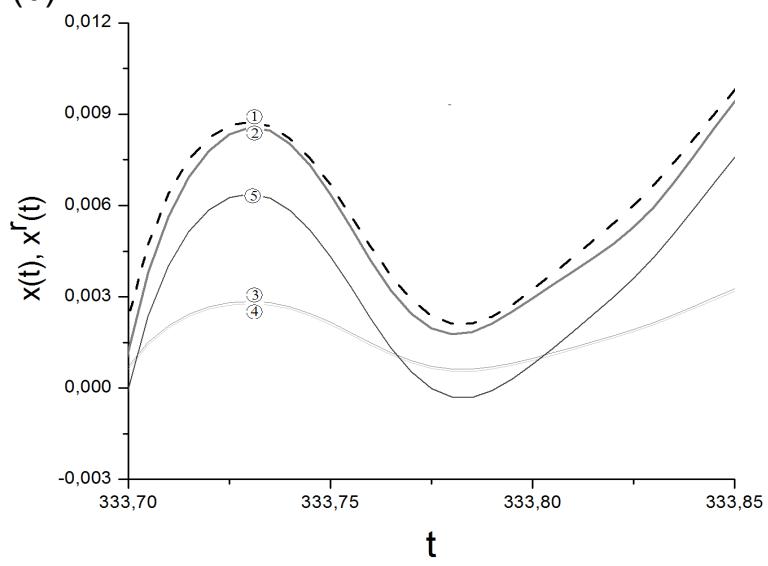

FIG. 5: Comparison of the numerical solutions of reconstructed flow $x^{r}(t)$ according to Eq.(11) with the original flow of Eq.(23) with weak and fast time periodic driving. The input is a superposition of $N=10$ oscillations defined by (51) where the amplitudes $I_{o}^{i}$ follow a Gaussian distribution with mean $\bar{w}_{o}=40$ and standard deviation $\sigma=30$. Panel (a) shows the original and reconstructed time series and panel $(b)$ is the focus onto the grey-shaded area in (a). The original flow (1, dashed curve) is compared to the reconstructed flow using a center manifold of cubic order expansion, and plotted with (2) and without (3) time-dependent correction. The trivial ansatz $h=0$ is also shown (4). In addition, (5) denotes the flow of Eq. (23) for $\tau=0$. We observe that the reconstructed flow with time-dependent center manifold follows the original system with great accuracy. Parameters are $I_{o}^{1}=0.1839, I_{o}^{2}=0.2569, I_{o}^{3}=0.3582, I_{o}^{4}=0.5, I_{o}^{5}=0.3582, I_{o}^{6}=0.2569, I_{o}^{7}=0.1839, I_{o}^{8}=0.1317$, $I_{o}^{9}=0.0944, I_{o}^{10}=0.676, w_{o}^{n}=10 \cdot n, n=1, \ldots, N, \tau=2.0, \varepsilon=0.2$ and $R_{2}=1.5$.

deterministic input above, the autonomous component of the center manifold is calculated using the very same procedure. However, in contrast to the previous discussion, the time-dependent correction (46) is now a solution of Eq. (45) if $H$ satisfies the linear delayed Langevin equation

$$
\frac{d H(t)}{d t}=-H(t)+H(t-\tau)+\kappa \xi(t) .
$$

Assuming initial conditions $H(r)=0,-\tau \leq r \leq 0$, the solution of (52) is given by [44]

$$
H(t)=\int_{0}^{t} H_{0}(t-s) d W(s)
$$

where $d W(t)$ is the differential of the Brownian motion coresponding to the random fluctuations $\xi(t)$ and where $H_{0}$ is the homogeneous solution of (52) for $\kappa=0$, given by

$$
H_{0}(t)=\frac{1}{2 \pi(1+\tau)}+\sum_{n=1}^{\infty} \frac{\exp \left(\lambda_{n} t\right)}{1+\tau \exp \left(-\lambda_{n} \tau\right)}+c . c .
$$

for initial conditions $H(\theta)=0,-\tau \leq \theta \leq 0$. The solution $H(t)$ is not stationary in the sense that its distribution function is not invariant with respect to time [44]. Moreover $H_{0}(t)=\sum_{n} c_{n} \exp (\lambda t)$, as discussed in the previous section. For this specific case, the dynamics on the slow manifold obeys

$$
\begin{array}{r}
\frac{d u(t)}{d t}=\frac{1}{1+\tau}\left(-\varepsilon\left(u+h_{\text {auto }}(-\tau, u)+h_{t}(-\tau, t)\right)-R_{2}\left(u+h_{\text {auto }}(-\tau, u)+h_{t}(-\tau, t)\right)^{2}+I(t)\right) \\
h_{t}(-\tau, t)=\kappa \int_{0}^{t-\tau} H_{0}(t-\tau-s) d W(s)-\frac{\kappa}{1+\tau} \int_{0}^{t} H_{0}(t-s) d W(s)-\frac{\kappa}{1+\tau} \int_{-\tau}^{0} \int_{0}^{t+s} H_{0}(t+s-r) d W(r) d s .
\end{array}
$$

In Fig. 6 we compare a single solution trajectory obtained numerically from the original problem in Eq. (23) to the reconstructed path $x^{r}(t)=u(t)+h(u, t)$. The figure illustrates clearly that the proposed method allows a very good reconstruction of the stochastic path in spite of the presence of the broad frequency band.

/noindent To evaluate the quality of the reconstruction more generally, Fig. 7 shows the reconstruction error subjected to the delay value and noise strength. The figure reveals that the reconstruction error is smallest for small noise strength and small delays. 


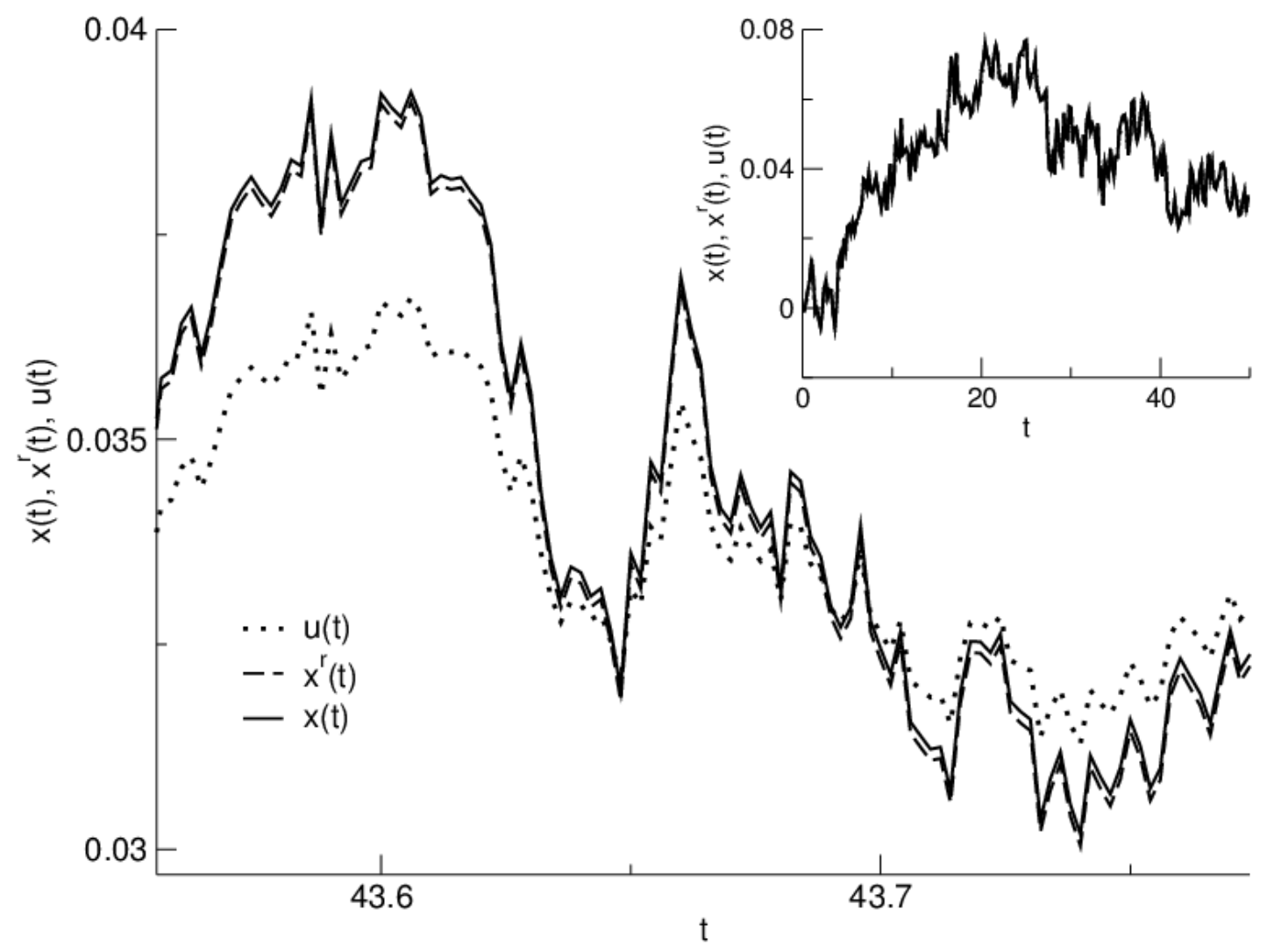

FIG. 6: Comparison of the numerical solutions of reconstructed flow $x^{r}(t)$ according to Eq.(11) with the original flow of Eq. (23) with white noise. The small inset shows a solution path $x(t)$ of the original equation (23), the reconstructed solutions $x^{r}(t)=u(t)+h_{2}(u)+h_{3}(u)+h_{t}(t)$ considering the full reduction method, and $u(t)$ alone neglecting the center manifold reduction. The large panel presents a focussed view of the solutions and reveals a very good approximation of the original solution by the reduced model solution. The numerical integration has been performed according to the delayed Euler-Maruyama scheme with time step $d t=0.001$. Parameters are $\tau=0.5, \epsilon=0.02, R_{2}=1.0, \kappa=0.01$. Initial conditions have been chosen as $x(\theta)=h_{2}(\theta, 0)+h_{3}(\theta, 0)=h_{t}(\theta)=0$ for $-\tau \leq \theta \leq 0$ and $u(0)=0$.

\section{Insights about stochastic systems stability}

The reduction method presented above constitutes a powerful method to analytically characterize the influence of time-fluctuating quantities on the dynamics and stability of delayed systems. To learn more about the effect of additive noise on the system, we perform a brief numerical investigation of the system. The system exhibits an unstable fixed point at $x_{u}=0$ for $\varepsilon<0$ and $x_{u}=-\varepsilon / R_{2}$ for $\varepsilon \leq 0$. We consider the probability density function of the stochastic process in the basin of attraction of the stable fixed point and, to this end, introduce an absorbing barrier at $x=x_{u}$ and $u=x_{u}$. Figure 8 shows the stationary probability density function (PDF) of $x(t)$ and $u(t)$ for different noise strengths $\kappa$ and values of $\varepsilon$. Based on the accuracy of the reconstruction discussed in previous sections, the PDF of both processes are in very good accordance for all parameters.

The figure also reveals a novel shift of the PDF to larger amplitudes with increased strength of the additive white noise on a transcritical bifurcation. Such an effect has been observed previously in scalar non-delayed systems in the presence of multiplicative noise only [26, 27, 45-48]. However, an additive noise-induced transition had been found numerically in a scalar DDE near a Hopf bifurcation [10, 49]. And recently, we have found that this also occurs at a pitchfork bifurcation in a delayed system [29]. Our method thus offers a novel analytical approach to characterize noise-induced shifts in DDE's by explicit treatment of the system's non-linearities and delayed components. 
(a)

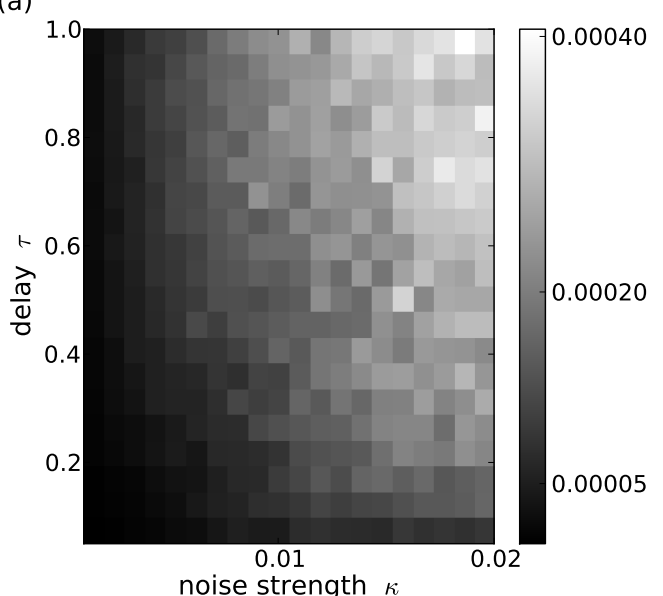

(b)

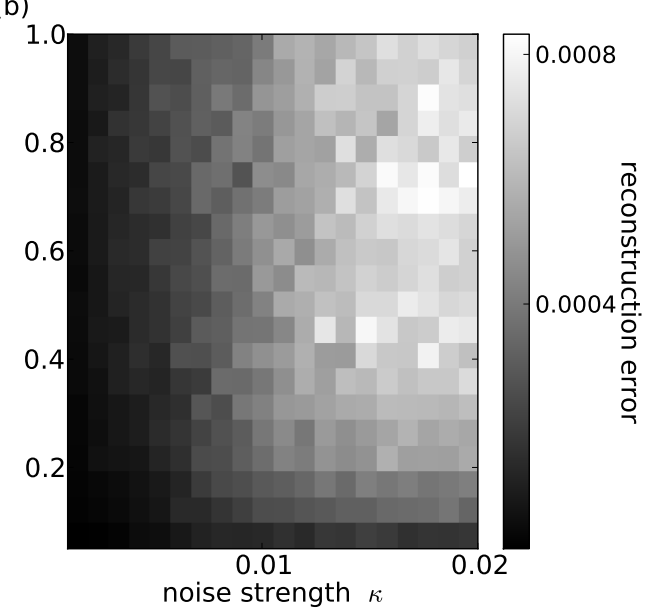

FIG. 7: Reconstruction error of the method for random inputs and various time delays and noise strengths. The reconstruction error is defined as $\sum_{i=1}^{T}\left|x\left(t_{i}\right)-x_{r}\left(t_{i}\right)\right| / T$ with the original solution $x(t)$ gained from Eq. (23) and the reconstructed solution $x^{r}(t)=u(t)+h(u, t)$ obtained from Eqs. (54), (55). Since the solutions are non-stationary in time, the mean error is computed as the ensemble average over 100 trajectories at an early $((\mathrm{a}), t=20 \tau)$ and a late $((\mathrm{b}), t=60 \tau)$ time instant. The other parameters are identical to the one in Fig. 6.

\section{DISCUSSION}

In this paper, we first summarized for a physics audience the procedure outlined in e.g. [30, 31, 34] by which an autonomous non-linear delay differential equation can be projected onto its center manifold. This produces an accurate non-delayed system reproducing the behavior of the original system in the vicinity of an instability. We then showed that the dynamics of a non-autonomous delayed feedback system could also be captured by center manifold reduction. This is made possible by allowing an explicit time-dependence of the center manifold, taking the form of an additive time-dependent correction to the non-driven problem. This term is also considered small and of quadratic order. The time-dependent center manifold is found to obey a linear non-homogeneous delay differential equation.The proposed time-dependent center manifold reduction scheme thus yields a low-dimensional non-delayed order parameter equation from the infinite-dimensional delay differential equation near a codimension-1 bifurcation with only one eigenvalue having zero real part.

We illustrated the approach by considering a scalar delay differential equation with quadratic non-linearity, driven by an additive time-periodic term, in the vicinity of a transcritical bifurcation. Numerical experiments support the analytical approach, especially for faster and weaker forcing, since they show good agreement of the reconstructed flow (to both quadratic and cubic order) with the dynamics of the original system with time-periodic driving. These reconstructions were clearly superior to the approximation based on the usual time-independent CM. This was the case also for multiple periodic inputs. The proposed method considerably reduces the error of the center manifold reduction when external driving is present by adjusting both the phase and the amplitude of the flow of the order parameter equation, thus yielding an accurate match to the original system's response. The method in fact accounts for the effects of the forcing on the nonlinear part of the system. An approach where the CM is first computed without forcing, then used to approximate the dynamics on the CM augmented with external forcing, misses these nonlinear effects, even though it may give acceptable results in some applications. We have also found good agreement for a delayed system with a pitchfork bifurcation (i.e. a cubic nonlinearity) (not shown).

Alhought the initial conditions of the reconstructed flow and those of the original system were made equal at $t=0$, it is still unclear how the history $[-\tau, 0)$ of the delayed system is mapped to the initial conditions of the OPE, which is non-delayed. Indeed, deviations between the evolution of both systems were apparent in the transient regime, but decrease as time increases, indicating that the approximations used in the analysis hold only for steady state regimes. A tentative solution to this problem would require a consideration of the initial value problem of the individual stable modes, an information that appears to be lost with the application of the center manifold theorem and following approximations. In addition, higher order expansions in both modes and time might be required to approach the early dynamics of the initial delayed problem. 
(a)

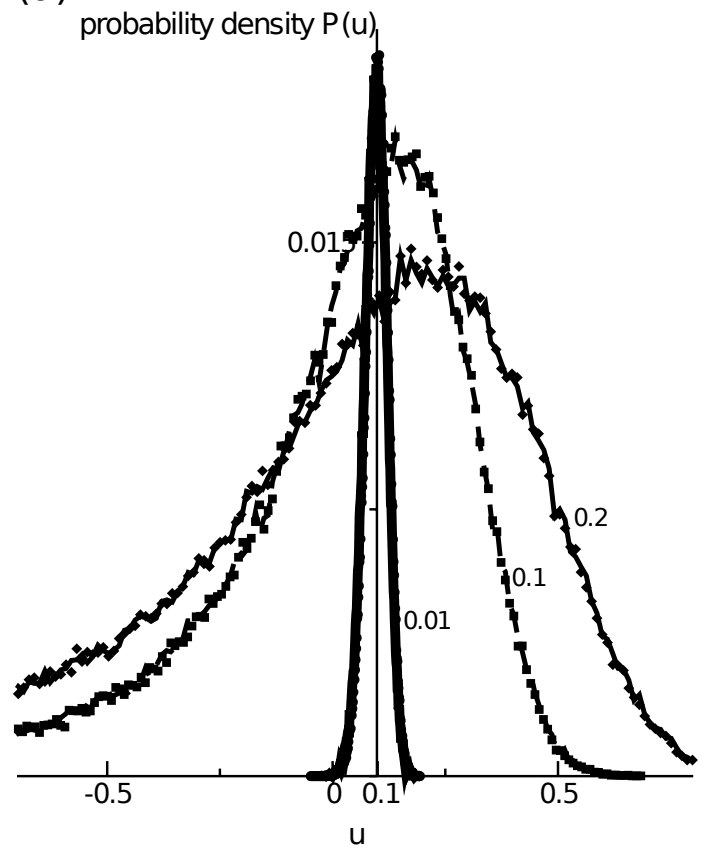

(b)

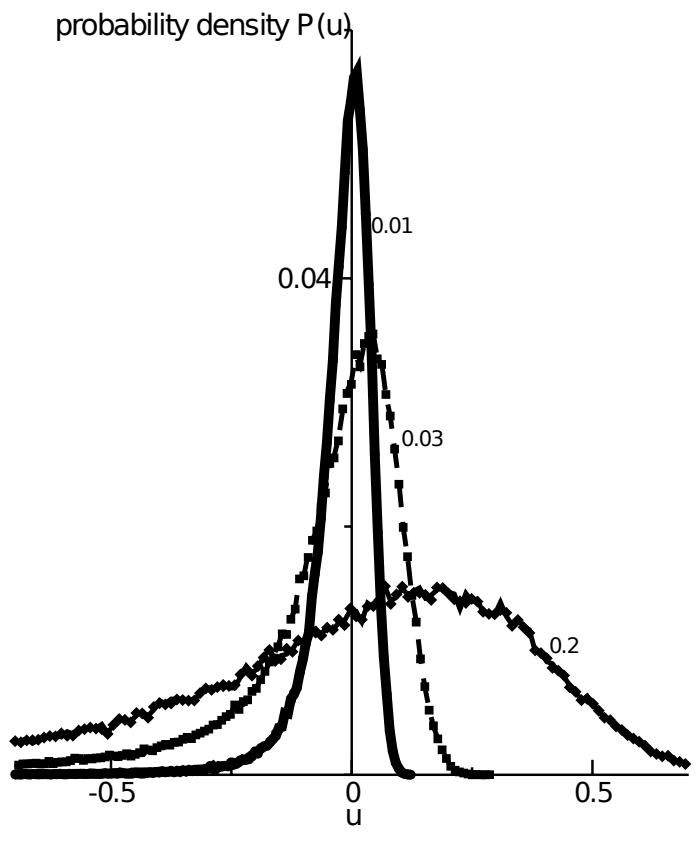

FIG. 8: Additive noise moves the probability density function of the delayed system to higher amplitudes. The probability density functions are given for the original system (lines) and order parameter equation (dots) which are in very good agreement. Parameters are (a) $\varepsilon=-0.1, \kappa=0.01$ (thick solid line), $\kappa=0.1$ (dashed thin line), $\kappa=0.2$ (thin line); (b) $\varepsilon=0.02, \kappa=0.01$ (thick solid line), $\kappa=0.03$ (dashed thin line) and $\kappa=0.2$ (thin line). The probability has been estimated by $15 \cdot 10^{4}$ trials at $t=5$. Other parameters are $\tau=0.05, R_{2}=1.0$ with the initial conditions (a) $x(\theta)=u(0)=0.1,(\mathrm{~b}) x(\theta)=u(0)=0.0$ and $h_{2}(\theta, 0)+h_{3}(\theta, 0)=h_{t}(\theta)=0$ in both cases for $-\tau \leq \theta \leq 0$. To ensure the existence of probability density functions, we have introduced absorbing barriers at $x=x u$ and $u=x u$.

While most of the work reported here pertains to the reduced dynamics in the presence of deterministic forcing, we have also considered the infinite frequency white noise input. This allowed us to bridge the deterministic results here to our recent work where the non-autonomous part is a stochastic forcing term driving a system at a pitchfork bifurcation [29]. In fact, that stochastic work now lies on firmer ground after the foregoing deterministic time-dependent CM analysis. Here as in that work, the method works best for small noise and delays - and the noise constraint is weakened by a smaller delay. It might be possible here to perform an approximate Fokker-Planck analysis on the stochastic center manifold equation as in [29] to quantify the shift in the maxima of the PDF for the transcritical case. In either case however, it is of interest to extend the method in order to relax the constraints, to see if e.g. it is somehow possible to treat the larger noise and delay cases without having to perform the tedious computations of higher terms on the center manifold.

Also, it would be highly interesting to extend the method to problems known to have a two-dimensional center manifold, those exhibiting a Hopf bifurcation in particular. Finally, our analysis required the external forcing with a single or multiple frequencies to be on a faster time scale than the slow bifurcating modes. For a given time scale of forcing, this condition can always be met by restraining the dynamics sufficiently close to the bifurcation point. Yet one would like to relax the constraint in order to generalize the method to arbitrary time scales of forcing.

\section{ACKNOWLEDGMENTS}

The authors we would like to thank NSERC and FQRNT for support. In addition Axel Hutt has received funding from the European Research Council under the European Union's Seventh Framework Programme (FP7/2007-2013)/ 
ERC grant agreement no. 257253. 
[1] R. Lang and K. Kobayashi. External optical feedback effects on semi-conductor injection laser properties. IEEE J. Quantum Electron., 16:347, 1980.

[2] T. Erneux. Applied Delay Differential Equations. Surveys and Tutorials in the Applied Mathematical Sciences. Springer, 2009.

[3] I.Z. Kiss, C.G. Rusin, H. Kori, and J.L. Hudson. Engineering complex dynamical structures: Sequential patterns and desynchronization. Science, page 1886, 2007.

[4] V. Kolmanovskii and A. Myshkis. Applied Theory of Functional Differential Equations. Kluwer, Boston, 1992.

[5] P. Hovel and E. Scholl. Control of steady states by time-delayed feedback methods. Phys. Rev. E, page $046203,2003$.

[6] J. Bechhoefer. Feedback for physicists: A tutorial essay on control. Reviews of Modern Physics, 77, 2005.

[7] F.M. Atay, editor. Complex Time-Delay Systems. Theory and Application. Springer, Berlin, 2010.

[8] N. MacDonald. Biological Delay Systems: Linear Stability Theory. ambridge Studies in Mathematical Biology Vol.8. Cambridge University Press, Cambridge, UK, 1989.

[9] L. Glass, A. Beuter, and D. Larocque. Time delays, oscillations, and chaos in physiological control systems. Math. Biosci., 90:111, 1988.

[10] A. Longtin, J.G. Milton, J. Bos M.C., and Mackey. Noise and critical behavior of the pupil light reflex at oscillation onset. Phys. Rev. A, 41:6992, 1990.

[11] C.W. Eurich and J.G. Milton. Noise-induced transitions in human postural sway. Phys. Rev. E, 54:6681-6684, 1996.

[12] J. Boulet, R. Balasubramaniam, A. Daffertshofer, and A. Longtin. Stochastic two delay-differential model of delayed visual feedback effects on postural dynamics. Phil. Trans. Royal Soc. A, 2009. in press.

[13] J. Belair, S.A. Campbell, and P. van den Driessche. Frustration, stability and delay-induced oscillations in a neural network model. S.I.A.M. Journal on Applied Mathematics, 56:245-255, 1996.

[14] V. K. Jirsa. Connectivity and dynamics of neural information processing. Neuroinformatics, pages 183-204, 2004.

[15] G. Deco, V. Jirsa, A.R. McIntosh, O. Sporns, and R. Kotter. Key role of coupling, delay, and noise in resting brain fluctuations. PNAS, 106:10302-10307, 2009.

[16] F.M. Schneider M.A. Dahlem and E. Scholl. Failure of feedback as a putative common mechanism of spreading depolarizations in migraine and stroke. Chaos, 18:026110, 2008.

[17] A. Hutt. Sleep and anesthesia. Frontiers in Neuroscience, 3(3):408-409, 2009.

[18] C. Laing and S. Coombes. The importance of different timings of excitatory and inhibitory pathways in neural field models. Network, 17, 151 (2006).

[19] A. Hutt and F.M. Atay. Neural fields with distributed transmission speeds and long-range feedback delays. SIAM Journal Applied Dynamical Systems, 5(4):670-698, 2006.

[20] J. Lefebvre, A. Longtin, and V.G. LeBlanc. Dynamics of driven recurrent networks of on and off cells. Phys. Rev. E, 80:041912, 2009.

[21] M.J. Chacron, A. Longtin, and L. Maler. Delayed excitatory and inhibitory feedback shape neural information transmission. Phys. Rev. E, 72:051917, 2005.

[22] S.-A. Campbell and J. Belair. Analytical and symbolically-assisted investigations of hopf bifurcations in delay-differential equations. Canadian Applied Mathematics Quarterly, 3:137-154, 1995.

[23] H. Haken. Synergetics - An Introduction. Springer, third and enlarged edition edition, 1983.

[24] C. Chicone and Y. Latushkin. Center manifolds for infinite dimensional nonautonomous differential equations. Journal of differential equations, 141:356-399, 1997.

[25] P. Boxler. A stochastic version of center manifold theory. Probab. Theory Relat. Fields, 83:509, 1989.

[26] D. Bloemker. Amplitude equations for locally cubic non-autonomous nonlinearities. SIAM J.Appl.Dyn.Syst., 2003.

[27] A. Hutt, A. Longtin, and L. Schimansky-Geier. Additive global noise delays turing bifurcations. Phys. Rev. Lett., 98(230601), 2007.

[28] S.M. Cox and A.J. Roberts. Center manifolds of forced dynamical systems. J. Austral. Math. Soc. Ser. B, 32:401-436, 1991.

[29] A. Hutt, J. Lefebvre, and A. Longtin. Delay stabilizes stochastic systems near a non-oscillatory instability. Europhys. Lett., 2012. in press.

[30] J.K. Hale and S.M.V. Lunel. Introduction to functional differential equations. Springer, Berlin, 1993.

[31] W. Wischert, A. Wunderlin, and A. Pelster. Delay-induced instabilities in nonlinear feedback systems. Physical Review E, 49(1), 1994.

[32] R. Quesmi, M. Ait Babram, and M.L. Hbid. A maple program for computing a terms of a center manifold, and element of bifurcations for a class of retarded functional differential equations with hopf singularity. Applied Mathematics and Computation, 175:932-968, 2006.

[33] S.-A. Campbell. Delay Differential Equations: Recent Advances and New Directions, chapter Calculating Center Manifolds for Delay Differential Equations Using Maple. Spinger-Verlag, New York, 2008.

[34] T. Faria and L. T. Magalhaes. Normal forms for retarded functional differential equations with parameters and applications to hopf bifurcation. Journal of Differential Equations, 122:281, 1995.

[35] M. Schanz and A. Pelster. Synergetic system analysis for the delay-induced hopf bifurcation in the wright equation. SIAM J. Applied Dynamical Systems, 2(3):277-296, 2003.

[36] R. Quesmi, M. Ait Babram, and M.L. Hbid. Center manifolds and normal forms for a class of retarded functional differential 
equations with parameter associated with fold-hopf singularity. Applied Mathematics and Computation, 181:220-246, 2006.

[37] F.M. Asl and A.G. Ulsoy. Analysis of a system of linear delay differential equations. Journal of Dynamic Systems, Measurement, and Control, 125, 2003.

[38] J.C. Li and C.H. Hansen. Forced phase-locked response of a nonlinear system with time delay after hopf bifurcation. Chaos, Solitons and Fractals, 25:461-473, 2005.

[39] J. Xu and K.W. Chung. Effects of time delayed position feedback on a van der pol2̆013duffing oscillator. Physica D, 180:17-39, 2003.

[40] T. D. Frank and P. J. Beek. Stationary solutions of linear stochastic delay differential equations: Applications to biological systems. Phys. Rev. E, 64:021917, 2001.

[41] S. Guillouzic, I. L'Heureux, and A. Longtin. Small delay approximation of stochastic delay differential equation. Phys. Rev. E, 59(4):3970, 1999.

[42] A. Amann, E. Scholl, and W. Just. Some basic remarks on eigenmode expansions of time-delay dynamics. Physica A, 373:191-202, 2007.

[43] R. Bellmann and K.L. Cooke. Differential-Difference Equations. Academic Press, New York, 1963.

[44] U. Küchler and B. Mensch. Langevin stochastic differential equation extended by a time-delayed term. Stoch. Stoch. Rep., 40:23-42, 1992.

[45] A. Hutt, A. Longtin, and L. Schimansky-Geier. Additive noise-induced turing transitions in spatial systems with applications to neural fields and wsift-hohenberg equations. Physica D, 237:755-773, 2008.

[46] A. Hutt. Additive noise may change the stability of non-linear systems. Europhys. Lett., 84:1-4, 2008.

[47] M. Pradas, D. Tseluiko, S. Kalliadasis, D.T. Papageorgiou, and G.A. Pavliotis. Noise induced state transitions, intermittency, and universality in the noisy kuramoto-sivashinksy equation. Phys. Rev. Lett., 106:060602, 2011.

[48] M. Gaudreault, F. Drolet, and J . Vinals. Analytical determination of the bifurcation thresholds in stochastic differential equations with delayed feedback. Phys. Rev. E, 82:051124, 2010.

[49] A. Longtin. Noise-induced transitions at a hopf bifurcation in a first order delay-differential equation. Phys. Rev. A, 44:4801, 1991.

[50] N. Yeganefar, P. Pepe, and M. Dambrine. Input-to-state stability of time-delay systems : A link with exponential stability. IEEE Transactions On Automatic Control, 53:1526-1531, 2008.

[51] J. Carr. Applications of Center Manifold Theory. Applied Mathematical Sciences 35. Springer verlag, New York, 1981.

[52] O. Arino, M.L. Hbid, and E. Ait Dads. Delay Differential Equations and Applications. NATO science series. Springer Verlag, 1999.

[53] J. Guckenheimer and P. Holmes. Nonlinear Oscillations, Dynamical Systems and Bifurcation of Vector Fields. Applied Mathematical Sciences 42. Spinger Verlag, 1983.

[54] B. Redmond, V.G. LeBlanc, and A . Longtin. Bifurcation analysis of a class of first-order nonlinear delay-differential equations with reflectional symmetry. Physica D, 166:131-146, 2002.

[55] G. Orosz. Hopf bifurcation calculations in delayed systems. Periodica Polytechnica Ser. mech. Eng., 48(2):198-200, 2004.

[56] X.-P. Yan and W.-T. Li. Hopf bifurcation and global periodic solutions in a delayed predator-prey system. Applied Mathematics and Computation, 177:427-445, 2006.

[57] S.A. Campbell et al. Limit cycles, tori and complex dynamics in a second-order differential equation with delayed negative feedback. J. Dyn. Diff. Eq., 7, 213 (1995).

[58] M.C. Mackey and L. Glass. Oscillation and chaos in physiological control systems. Science, 197:287-289, 1977.

[59] C. Koch. Biophysics of Computation. Oxford University Press, Oxford, 1999.

[60] A. Hutt and F.M. Atay. Analysis of nonlocal neural fields for both general and gamma-distributed connectivities. Physica $D, 203: 30-54,2005$.

[61] A. Hutt. Effects of nonlocal feedback on traveling fronts in neural fields subject to transmission delay. Phys. Rev. E, 70:052902, 2004.

[62] Brian N. Pasley and Elena A. Allen and Ralph D. Freeman State-Dependent Variability of Neuronal Responses to Transcranial Magnetic Stimulation of the Visual Cortex. Neuron, 62, 291-303, 2009

[63] Herrmann CS Human EEG responses to 1-100 Hz flicker: resonance phenomena in visual cortex and their potential correlation to cognitive phenomena. Exp Brain Res., 137, 346-53, 2001

[64] Tsang EW and Hamani C and Moro E and Mazzella F and Saha U and Lozano AM and Hodaie M and Chuang R and Steeves T and Lim SY and Neagu B and Chen R. Subthalamic deep brain stimulation at individualized frequencies for Parkinson disease. Neurology, 78, 1930-8, 2012 